\title{
Centroid theory of transverse electron-proton two-stream instability in a long proton bunch
}

\author{
Tai-Sen F. Wang, Paul J. Channell, and Robert J. Macek \\ Los Alamos National Laboratory, P.O. Box 1663, Los Alamos, New Mexico 87545 \\ Ronald C. Davidson \\ Plasma Physics Laboratory, Princeton University, Princeton, New Jersey 08543
}

(Received 15 September 2002; published 22 January 2003)

\begin{abstract}
This paper presents an analytical investigation of the transverse electron-proton $(e-p)$ two-stream instability in a proton bunch propagating through a stationary electron background. The equations of motion, including damping effects, are derived for the centroids of the proton beam and the electron cloud by considering Lorentzian and Gaussian frequency spreads for the particles. For a Lorentzian frequency distribution, we derive the asymptotic solution of the coupled linear centroid equations in the time domain and study the $e$ - $p$ instability in proton bunches with nonuniform line densities. Examples are given for both uniform and parabolic proton line densities.
\end{abstract}

DOI: 10.1103/PhysRevSTAB.6.014204

PACS numbers: 29.27.Bd, 41.75.-i, 52.40.Mj, 52.35.Qz

\section{INTRODUCTION}

The transverse two-stream instability has been of longstanding interest in research involving beam propagation through a charged particle background. For acceleratorgenerated intense proton beams, the recent focus of twostream efforts has been on the transverse instability observed in the Proton Storage Ring (PSR) at Los Alamos [1-24]. The PSR stores a long proton bunch with a near triangular line density profile for a typical duration of about $1 \mathrm{~ms}$. Depending on machine conditions, the instability is observed as rapidly growing transverse oscillations of the stored beam, usually occurring when the beam intensity reaches about $2.5 \times 10^{13}$ protons per pulse or higher, causing fast beam loss. Over the years, experimental evidence has been collected to support the conjecture that the instability in PSR is due to the interaction between the circulating proton beam and electrons created in the ring, the electron-proton $(e-p)$ instability. However, the understanding of the instability is still based on the theory developed for a continuous beam with uniform line density [15-29]. Although computer simulations have been implemented or are being developed $[12,15,16,19,20]$ to study the $e$ - $p$ instability in bunched beams, a companion analytical theory remains to be developed.

The present work is an attempt to investigate the transverse $e-p$ instability in a proton bunch using an analytical approach based on the centroid model. Because recent experiments at PSR have demonstrated that most of the electrons in the ring are created during the passage of the proton bunch and are lost to the beam pipe afterward [7-10,14], the present model is built on the assumption of a "one-pass" interaction between the proton beam and the background electrons; that is, in the proton beam frame, electrons pass through the proton bunch only once. In the following, we derive the equations of motion for the centroids of the proton beam and the background electron cloud. The damping effect is incorporated by considering a Lorentzian distribution or a Gaussian distribution in the betatron frequency of the protons and the bounce frequency of the electrons in the proton beam field (see Sec. II). For a Lorentzian distribution of particle frequencies, a general formalism for obtaining the asymptotic solution of the coupled centroid equations in the time domain is developed and used to study the instability of a proton bunch propagating through a stationary, nonuniform electron background. The theoretical model is described in Sec. II, and the growth rate of the $e-p$ instability is discussed and a dispersion relation is derived for the case of uniform electron background in Sec. III. Examples are also given for proton line densities with uniform and parabolic profiles in Sec. IV.

\section{THEORETICAL MODEL}

We consider a bunched proton beam with full length $L$ and circular cross section of radius $a$, propagating with a constant velocity $\mathbf{v}$ through a stationary electron background of infinite extent in the direction of beam propagation. A Cartesian coordinate system is adopted such that the $z$ axis is pointing opposite to the direction of proton propagation, and the $y$ axis is perpendicular to the ring. The origin of the coordinate system is chosen to coincide with the center of the beam cross section. We assume that in the equilibrium state, the electrons are trapped in the proton beam, and both species of particles are distributed uniformly in the transverse direction so that the trapped electrons experience a linear transverse focusing force. However, the line densities of the protons and electrons, $\lambda_{p}$ and $\lambda_{e}$, are allowed to depend on $z$. The protons are focused in the transverse direction by a force that depends linearly on the radial displacement of the particles. For simplicity, the synchrotron motion of the protons and the axial motion of the electrons in the 
laboratory frame are neglected. Further simplifications result from neglecting the impedance due to the beam environment, such as the beam pipe, and from considering the transverse motion in only one direction, say, the $y$ direction. The proton bunch considered is "long" in the sense that an electron will execute many transverse bounces between the time it enters the proton bunch at the head and the time it exits at the tail of the proton bunch. Our stability study is focused on the one-pass interaction between the proton bunch and the electrons; that is, the proton bunch is traveling in a stationary electron background of infinite extent in the axial direction so that the electrons interact with the proton beam only once. As mentioned earlier, for the $e-p$ instability in PSR, this kind of model should be more realistic than one with periodic interactions between the protons and the electrons.

This investigation of the $e-p$ instability will be based on analyzing the motions of the centroid of the proton beam $Y_{p}=Y_{p}(z, t)$ and the centroid of the trapped electrons $Y_{e}=Y_{e}(z, t)$ defined by

$$
Y_{p}(z, t)=\int_{-\infty}^{\infty} y_{p}\left(z, t, \omega_{p}\right) F_{p}\left(\omega_{p}\right) d\left(\omega_{p} / \Delta_{p}\right),
$$

and

$$
Y_{e}(z, t)=\int_{-\infty}^{\infty} y_{e}\left(z, t, \omega_{e}\right) F_{e}\left(\omega_{e}\right) d\left(\omega_{e} / \Delta_{e}\right),
$$

where the subscripts $p$ and $e$ denote the protons and electrons, respectively; $y_{p}\left(z, t, \omega_{p}\right)$ and $y_{e}\left(z, t, \omega_{e}\right)$ are the displacements in the $y$ direction at position $z$ and time $t ; \omega_{p}$ and $\omega_{e}$ are the oscillation frequencies; $F_{p}\left(\omega_{p}\right)$ and $F_{p}\left(\omega_{p}\right)$ are the frequency distribution functions; and $\Delta_{p}$ and $\Delta_{e}$ are quantities that characterize the spreads of $\omega_{p}$ and $\omega_{e}$, respectively.

The centroids defined in Eqs. (1) and (2) allow us to incorporate the effects of damping of the centroid motion due to the frequency spreads. To derive the equations of motion of the centroids, we first neglect the axial motion of the particles and consider the equations of motion for single particles. This gives

$$
\frac{d^{2} y_{p}}{d t^{2}}=-\omega_{p}^{2} y_{p}+\frac{F_{p}^{\mathrm{per}}}{\gamma m_{p}},
$$

and

$$
\frac{d^{2} y_{e}}{d t^{2}}=-\omega_{e}^{2} y_{e}+\frac{F_{e}^{\mathrm{per}}}{m_{e}},
$$

where $F_{p}^{\mathrm{per}}$ is the perturbing force on a proton due to the displaced electron cloud, $F_{e}^{\mathrm{per}}$ is the perturbing force on an electron due to the displaced proton beam, $\omega_{p}^{2} y_{p}$ and $\omega_{e}^{2} y_{e}$ are the linear forces, $m_{p}$ and $m_{e}$ are the rest mass of a proton and an electron, respectively, $\gamma=$ $\left(1-v^{2} / c^{2}\right)^{-1 / 2}$ is the relativistic mass factor, and $c$ is the speed of light in vacuum. The general solutions to Eqs. (3) and (4) can be expressed as

$$
y_{p}\left(z, t, \omega_{p}\right)=A_{p} e^{i \omega_{p} t}+A_{p}^{*} e^{-i \omega_{p} t},
$$

and

$$
y_{e}\left(z, t, \omega_{e}\right)=A_{e} e^{i \omega_{e} t}+A_{e}^{*} e^{-i \omega_{e} t},
$$

where $i=\sqrt{-1}$, the superscript $*$ denotes the complex conjugate, and the amplitudes $A_{p}$ and $A_{e}$ are assumed to depend on $z$ and $t$ but not on the single-particle oscillation frequency. The centroid equations are derived by considering a specific type of frequency distribution function. In this paper, we focus on the Lorentzian distribution functions

$$
F_{p}\left(\omega_{p}\right)=\frac{\Delta_{p}^{2}}{\pi\left[\Delta_{p}^{2}+\left(\omega_{p}-\omega_{p o}\right)^{2}\right]}
$$

and

$$
F_{e}\left(\omega_{e}\right)=\frac{\Delta_{e}^{2}}{\pi\left[\Delta_{e}^{2}+\left(\omega_{e}-\omega_{e o}\right)^{2}\right]},
$$

where $\omega_{p o}$ and $\omega_{e o}$ are the central oscillation frequencies of the protons and electrons, respectively.

We derive the centroid equation for the protons as an illustration of the procedure. The centroid equation for the electrons can be derived using a similar approach. First, applying the residue theorem of complex integrations, we calculate the derivative of $Y_{p}$ according to

$$
\begin{aligned}
\frac{d Y_{p}}{d t}= & \frac{1}{\pi} \int_{-\infty}^{\infty} \frac{\Delta_{p} d y_{p} / d t}{\Delta_{p}^{2}+\left(\omega_{p}-\omega_{p o}\right)^{2}} d \omega_{p} \\
= & i e^{-\Delta_{p} t}\left[A_{p}\left(\omega_{p o}+i \Delta_{p}\right) e^{i \omega_{p o} t}\right. \\
& \left.-A_{p}^{*}\left(\omega_{p o}-i \Delta_{p}\right) e^{-i \omega_{p o} t}\right] .
\end{aligned}
$$

The average of $\omega_{p}^{2} y_{p}$ is then evaluated using the above results as

$$
\begin{aligned}
\int_{-\infty}^{\infty} \omega_{p}^{2} y_{p}\left(z, t, \omega_{p}\right) F_{p}\left(\omega_{p}\right) d\left(\omega_{p} / \Delta_{p}\right) \\
=\frac{1}{\pi} \int_{-\infty}^{\infty} \frac{\Delta_{p} \omega_{p}^{2} y_{p}}{\Delta_{p}^{2}+\left(\omega_{p}-\omega_{p o}\right)^{2}} d \omega_{p} \\
=\left(\omega_{p o}^{2}+\Delta_{p}^{2}\right) Y_{p}+2 \Delta_{p} \frac{d Y_{p}}{d t} .
\end{aligned}
$$

Applying Eqs. (9) and (10), the average of Eq. (3) over frequency can be carried out readily to yield

$$
\frac{d^{2} Y_{p}}{d t^{2}}+2 \Delta_{p} \frac{d Y_{p}}{d t}+\left(\omega_{p o}^{2}+\Delta_{p}^{2}\right) Y_{p}=\frac{F_{p}^{\text {per }}}{\gamma m_{p}},
$$

where we have made the assumption that $F_{p}^{\text {per }}$ is independent of $\omega_{p}$. Following the same procedure, it can be shown that

$$
\frac{d^{2} Y_{e}}{d t^{2}}+2 \Delta_{e} \frac{d Y_{e}}{d t}+\left(\omega_{e o}^{2}+\Delta_{e}^{2}\right) Y_{e}=\frac{F_{e}^{\mathrm{per}}}{m_{e}} .
$$

For the model studied here, we assume that the incoherent betatron frequency shift due to the self-field of the 
proton beam is negligible, and that the electron density is much lower than that of the protons. Accordingly, the central oscillation frequency of the protons $\omega_{p o}$ is approximately the undepressed betatron oscillation frequency $\omega_{\beta}$, and the central oscillation frequency of the electrons $\omega_{e o}$ is given by

$$
\omega_{e o}=\Omega(z)=(c / a) \sqrt{2 r_{e} \lambda_{p}(z)[1-f(z)]},
$$

where $\Omega(z)$ is the electron bounce frequency, $r_{e}=$ $e^{2} /\left(m_{e} c^{2}\right)$ is the classical electron radius, and $f(z)=$ $\lambda_{e}(z) / \lambda_{p}(z)$ is the fractional charge neutralization. To simplify the mathematical analysis, we make the approximation that the perturbing force $F_{p}^{\text {per }}$ depends linearly on the displacement of the electron centroid, i.e.,

$$
\frac{F_{p}^{\mathrm{per}}}{\gamma m_{p}}=G(z) Y_{e},
$$

where $G(z)$ is defined by

$$
G(z)=2 r_{p} c^{2} \lambda_{e}(z) /\left(a^{2} \gamma\right)=\omega_{\beta}^{2} \xi(z) .
$$

Here, $r_{p}=e^{2} /\left(m_{p} c^{2}\right)$ is the classical proton radius, and

$$
\xi(z)=2 r_{p} c^{2} \lambda_{e}(z) /\left(a^{2} \gamma \omega_{\beta}^{2}\right) .
$$

Similarly, $F_{e}^{\text {per }}$ is approximated by a linear force proportional to the displacement of the proton centroid, i.e.,

$$
\frac{F_{e}^{\mathrm{per}}}{m_{e}}=\Omega^{2}(z) Y_{p}
$$

Equations (14) and (17) are consistent with the approximation that the perturbed transverse field due to a small transverse displacement of a beam is roughly proportional to the displacement [30]. The same and the similar kind of approximations have been used in Refs. [26-29] for analyzing the $e-p$ instability, and for studying the transverse beam stability due to the impedance in accelerators [31].

For the traveling proton beam in the laboratory frame, the derivatives with respect to time in Eq. (11) are replaced by total derivatives (convective derivatives following the average particle motion). Therefore, in the laboratory frame we have

$$
\left(\frac{\partial}{\partial t}-v \frac{\partial}{\partial z}\right)^{2} Y_{p}+2 \Delta_{p}\left(\frac{\partial Y_{p}}{\partial t}-v \frac{\partial Y_{p}}{\partial z}\right)+\left(\omega_{\beta}^{2}+\Delta_{p}^{2}\right) Y_{p}=G(z) Y_{e},
$$

and

$$
\frac{d^{2} Y_{e}}{d t^{2}}+2 \Delta_{e} \frac{d Y_{e}}{d t}+\left[\Omega^{2}(z, t)+\Delta_{e}^{2}\right] Y_{e}=\Omega^{2}(z, t) Y_{p},
$$

where the minus sign is used in the terms containing $\partial / \partial z$ because the proton bunch is propagating in the negative $z$ direction. In the beam frame, the proton bunch is stationary and the background electrons are traveling with axial velocity $v$, so Eqs. (18) and (19) are transformed to

$$
\frac{d^{2} Y_{p}}{d t^{2}}+2 \Delta_{p} \frac{d Y_{p}}{d t}+\left(\omega_{\beta}^{2}+\Delta_{p}^{2}\right) Y_{p}=G\left(z^{\prime}, t\right) Y_{e},
$$

and

$$
\left(\frac{\partial}{\partial t}+v \frac{\partial}{\partial z^{\prime}}\right)^{2} Y_{e}+2 \Delta_{e}\left(\frac{\partial Y_{e}}{\partial t}+v \frac{\partial Y_{e}}{\partial z^{\prime}}\right)+\left[\Omega^{2}\left(z^{\prime}\right)+\Delta_{e}^{2}\right] Y_{e}=\Omega^{2}\left(z^{\prime}\right) Y_{p},
$$

where $z^{\prime}$ is the axial coordinate in the beam frame.

One should keep in mind that the centroid equations here are derived for the case of Lorentzian distributions in the oscillation frequencies. The damping effect as well as the centroid equations depends on the choice of distribution of oscillation frequencies. Had we chosen a Gaussian distribution function,

$$
F_{q}\left(\omega_{q}\right)=\frac{1}{\sqrt{2 \pi}} \exp \left[\frac{-\left(\omega_{q}-\omega_{q o}\right)^{2}}{2 \Delta_{q}^{2}}\right]
$$

where the subscript $q$ denotes either protons or electrons, the centroid equation for stationary protons or electrons would be

$$
\frac{d^{2} Y_{q}}{d t^{2}}+2 \Delta_{q}^{2} t \frac{d Y_{q}}{d t}+\left[\Delta_{q}^{2}\left(1+\Delta_{q}^{2} t^{2}\right)+\omega_{q o}^{2}\right] Y_{q}=F_{q}^{\mathrm{per}}
$$

instead of Eqs. (11) and (12). To see the resulting difference in the damping effects, one can assume an impulse perturbation with $F_{q}^{\text {per }} \sim \delta(t)$ in the centroid equations and derive the solution $Y_{q} \sim \exp \left[\left(-\Delta_{q} \pm i \omega_{q o}\right) t\right]$ for Eqs. (11) and (12), and the solution $Y_{q} \sim \exp \left(-\Delta_{q}^{2} t^{2} / 2 \pm\right.$ $\left.i \omega_{q o} t\right)$ for Eq. (23). Thus, for a Lorentzian frequency distribution, the exponential damping exponent is linear in time, while for a Gaussian frequency distribution, the exponential damping exponent is quadratic in time. The calculation involved in the stability analysis for Gaussian 
distributions is considerably more complex than that for Lorentzian distributions, and only limited cases have been treated analytically (e.g., see Ref. [32]). We therefore focus the subsequent analysis on the case of Lorentzian frequency distributions.

\section{STABILITY ANALYSIS}

Our subsequent study of the $e-p$ instability is based on Eqs. (18)-(21). Since these equations are linear in $Y_{p}$ and $Y_{e}$, the analysis is necessarily limited to the linear regime of instability development. As the study is not limited to the case of axially uniform charge densities, we adopt a time-domain approach for easy extraction of the important physics. We also briefly describe the frequencydomain approach by deriving a dispersion relation in Sec. III B. Furthermore, because the initial growth rate of the unstable motion is complicated and depends in detail on the initial perturbation, our attention will be focused on the long time asymptotic behavior of the unstable modes.

\section{A. Perturbed motion}

We use $z$ and $z^{\prime}$ to denote the axial coordinates in the laboratory and the beam frames, respectively. These two coordinate systems are oriented and synchronized in the way that the proton beam travels in the negative $z$ direction and the origins coincide at $t=0$. The head of the proton bunch is chosen to be the origin of the beam-frame coordinate system. For simplicity, relativistic effects are neglected and Galilean transformations are used between the two coordinate systems. With this assumption, time coordinates are the same in both systems and the axial positions in these two coordinate systems are related by $z^{\prime}=z+v t$ or $z=z^{\prime}-v t$. If we assume that the proton bunch has a sharp-edged line density, and that $t_{e}$ is the time when a slice of electrons located at position $z$ in the laboratory frame ( $z^{\prime}$ in the beam frame) enters the proton bunch, then $t_{e}=-z / v=t-z^{\prime} / v$, and $\Omega\left(t-t_{e}\right)=0$ when $t \leq t_{e}$.

We begin the stability analysis by substituting

$$
Y_{p}\left(z^{\prime}, t\right)=e^{-\Delta_{p} t} Y_{p n}\left(z^{\prime}, t\right)
$$

and

$$
Y_{e}(z, t)=e^{-\Delta_{e} t} Y_{e n}(z, t),
$$

into Eqs. (20) and (19), respectively, to remove the damping terms from the left-hand sides of the equations of motion. This transforms the centroid equations to

$$
\frac{d^{2} Y_{p n}}{d t^{2}}+\omega_{\beta}^{2} Y_{p n}=G\left(z^{\prime}\right) e^{\left(\Delta_{p}-\Delta_{e}\right) t} Y_{e n}
$$

and

$$
\frac{d^{2} Y_{e n}}{d t^{2}}+\Omega^{2}\left[v\left(t-t_{e}\right)\right] Y_{e n}=\Omega^{2}\left[v\left(t-t_{e}\right)\right] e^{-\left(\Delta_{p}-\Delta_{e}\right) t} Y_{p n}
$$

It will be shown in the following analysis that the solution of the homogeneous part of Eq. (27),

$$
\frac{d^{2} Y_{e n}}{d t^{2}}+\Omega^{2}\left[v\left(t-t_{e}\right)\right] Y_{e n}=0,
$$

which describes the electron motion in the equilibrium proton bunch, will determine the asymptotic spatial oscillation of the perturbed centroids. Denoting the two linearly independent solutions of Eq. (28) by $\Phi=\Phi(t)$ and $\Psi=\Psi(t)$, and using the variation of parameters method, we obtain in the laboratory frame

$$
Y_{e n}(z, t)=\int_{t_{e}}^{t} \frac{\Omega^{2}\left[v\left(s-t_{e}\right)\right]}{v W\left[v\left(s-t_{e}\right)\right]}\left\{\Phi\left[v\left(t-t_{e}\right)\right] \Psi\left[v\left(s-t_{e}\right)\right]-\Psi\left[v\left(t-t_{e}\right)\right] \Phi\left[v\left(s-t_{e}\right)\right]\right\} e^{\left(\Delta_{e}-\Delta_{p}\right) s} Y_{p n}(z+v s, s) d s,
$$

where

$$
W(x)=\frac{d \Phi(x)}{d x} \Psi(x)-\frac{d \Psi(x)}{d x} \Phi(x),
$$

is the Wronskian of $\Phi(x)$ and $\Psi(x)$. Here, $Y_{p n}\left(z^{\prime}, t\right)$ has been transformed to the laboratory-frame variables using $z^{\prime}=$ $z+v t$, and the initial condition $Y_{e n}\left(0, t_{e}\right)=d Y_{e n}(0, t) /\left.d t\right|_{t=t_{e}}=0$ has been assumed. One can prove that the Wronskian is a constant by taking the derivative of $W(x)$ and applying Eq. (28). Because we are looking for the asymptotic solution, the other initial conditions involving $Y_{e n}\left(z, t_{e}\right)$ and $d Y_{e n}(z, t) /\left.d t\right|_{t=t_{e}}$ as functions of $z$ have been ignored. Note that, since in our model each electron passes through the proton bunch only once, the upper limit of integration in Eq. (29), $t$, is bounded by $t \leq t_{e}+L / v$.

We carry out the remaining analysis in the beam frame. Thus, rewriting Eq. (29) in beam-frame variables using $z=z^{\prime}-v t$, we obtain

$$
Y_{e n}\left(z^{\prime}, t\right)=\frac{1}{v W} \int_{t-z^{\prime} / v}^{t} \Omega^{2}\left[v(s-t)+z^{\prime}\right]\left\{\Phi\left(z^{\prime}\right) \Psi\left[v(s-t)+z^{\prime}\right]-\Psi\left(z^{\prime}\right) \Phi\left[v(s-t)+z^{\prime}\right]\right\} e^{\left(\Delta_{e}-\Delta_{p}\right) s} Y_{p n}\left(z^{\prime}+v s-v t, s\right) d s .
$$

Changing variables from $s$ to $r$ defined by $r=t-s$ in Eq. (31), and substituting the result into Eq. (26), we obtain 


$$
\frac{d^{2} Y_{p n}}{d t^{2}}+\omega_{\beta}^{2} Y_{p n}=\frac{G\left(z^{\prime}\right)}{v W} \int_{0}^{z^{\prime} / v} \Omega^{2}\left(z^{\prime}-v r\right)\left[\Phi\left(z^{\prime}\right) \Psi\left(z^{\prime}-v r\right)-\Psi\left(z^{\prime}\right) \Phi\left(z^{\prime}-v r\right)\right] e^{\left(\Delta_{p}-\Delta_{e}\right) r} Y_{p n}\left(z^{\prime}-v r, t-r\right) d r .
$$

Applying the Fourier transformation,

$$
Y_{p n}\left(z^{\prime}, t\right)=\frac{1}{\sqrt{2 \pi}} \int_{-\infty}^{\infty} \tilde{Y}\left(z^{\prime}, \omega\right) e^{i \omega t} d \omega
$$

to Eq. (32) and changing variables again by letting $x=z^{\prime}-v r$, we derive an integral equation for $\tilde{Y}\left(z^{\prime}, \omega\right)$ given by

$$
\tilde{Y}\left(z^{\prime}, \omega\right)=\frac{G\left(z^{\prime}\right) e^{\left(\Delta_{p}-\Delta_{e}-i \omega\right) z^{\prime} / v}}{v^{2} W\left(\omega_{\beta}^{2}-\omega^{2}\right)} \int_{0}^{z^{\prime}} \Omega^{2}(x)\left[\Phi\left(z^{\prime}\right) \Psi(x)-\Psi\left(z^{\prime}\right) \Phi(x)\right] \tilde{Y}(x, \omega) e^{-\left(\Delta_{p}-\Delta_{e}-i \omega\right) x / v} d x .
$$

It should be noted here that by choosing the Fourier-transformation approach in Eq. (33) instead of doing a Laplace transformation, we are looking for the asymptotic solutions by neglecting the influence of initial conditions. Making the substitution

in Eq. (34) yields

$$
\tilde{Y}\left(z^{\prime}, \omega\right)=\hat{Y}\left(z^{\prime}, \omega\right) G\left(z^{\prime}\right) e^{\left(\Delta_{p}-\Delta_{e}-i \omega\right) z^{\prime} / v}
$$

$$
\hat{Y}\left(z^{\prime}, \omega\right)=\frac{1}{v^{2} W\left(\omega_{\beta}^{2}-\omega^{2}\right)} \int_{0}^{z^{\prime}} \Omega^{2}(x) G(x)\left[\Phi\left(z^{\prime}\right) \Psi(x)-\Psi\left(z^{\prime}\right) \Phi(x)\right] \hat{Y}(x, \omega) d x .
$$

We must now solve the integral Eq. (36), use the relation in Eq. (35), and then invert the Fourier transformation. The mathematical manipulations involved in that process are very complex. A useful approximation will result from substituting

$$
\hat{Y}\left(z^{\prime}, \omega\right)=\omega_{\beta}^{-3} \zeta\left(z^{\prime}, \omega\right) \Phi\left(z^{\prime}\right)
$$

into Eq. (36), which gives

$$
\zeta\left(z^{\prime}, \omega\right)=\frac{1}{v^{2} W\left(\omega_{\beta}^{2}-\omega^{2}\right)} \int_{0}^{z^{\prime}} \Omega^{2}(x) G(x) \Phi(x) \Psi(x)\left[1-\frac{\Psi\left(z^{\prime}\right) \Phi(x)}{\Phi\left(z^{\prime}\right) \Psi(x)}\right] \zeta(x, \omega) d x .
$$

We investigate only the cases in which $\Omega^{2}(x)$ and $G(x)$ are smooth functions of $x$. It is reasonable to assume the two independent solutions of Eq. (28), $\Phi(x)$ and $\Psi(x)$, which describe the electron bounce motion in the proton bunch, are oscillatory functions that can be expressed as

$$
\Phi(x)=R(x) e^{i \Theta(x)},
$$

and

$$
\Psi(x)=R(x) e^{-i \Theta(x)},
$$

where $\Phi(x) \Psi(x)=R^{2}(x)$ is a smooth function and $\Phi(x) / \Psi(x)=e^{2 i \Theta(x)}$ is a rapidly oscillatory function. We assume that $\zeta\left(z^{\prime}, \omega\right)$ primarily describes the betatron oscillations at much lower frequency than the electron bounce motion in the beam. Under these assumptions, one would expect the fast oscillatory term containing $\Phi(x) / \Psi(x)$ to contribute much less than the term containing 1 in the integrand in Eq. (38). Hence, neglecting the term $\Phi(x) / \Psi(x)$, Eq. (38) simplifies to

$$
\zeta\left(z^{\prime}, \omega\right) \approx \int_{0}^{z^{\prime}} \frac{\Omega^{2}(x) G(x) R^{2}(x)}{v^{2} W\left(\omega_{\beta}^{2}-\omega^{2}\right)} \zeta(x, \omega) d x,
$$

which leads to

$$
\zeta\left(z^{\prime}, \omega\right) \approx \exp \left[\frac{i \omega_{\beta}^{2} J\left(z^{\prime}\right)}{k^{2}-\omega_{\beta}^{2}}\right],
$$

and

$$
\begin{aligned}
\tilde{Y}\left(z^{\prime}, \omega\right) \approx \omega_{\beta}^{-3} \Phi\left(z^{\prime}\right) G\left(z^{\prime}\right) \exp [ & \left(\Delta_{p}-\Delta_{e}-i \omega\right) \frac{z^{\prime}}{v} \\
& \left.+\frac{i \omega_{\beta}^{2} J\left(z^{\prime}\right)}{\omega^{2}-\omega_{\beta}^{2}}\right] .
\end{aligned}
$$

Here,

$$
\begin{aligned}
J\left(z^{\prime}\right) & =\frac{i}{\left(v \omega_{\beta}\right)^{2} W} \int_{0}^{z^{\prime}} \Omega^{2}(x) G(x) \Phi(x) \Psi(x) d x \\
& =\frac{1}{2 v^{2}} \int_{0}^{z^{\prime}} \frac{\Omega^{2}(x) \xi(x)}{d \Theta(x) / d x} d x
\end{aligned}
$$

is a quantity that can be used to characterize the coupling between the proton beam and the background electrons. Because $\Theta\left(z^{\prime}\right)$ is essentially the cumulative phase advance of the electrons' spatial oscillation in the positive $z^{\prime}$ direction, it follows that $d \Theta(x) / d x$ is like a positive 
wave number. Therefore, $J\left(z^{\prime}\right)$ is expected to be a positive, monotonic increasing function of $z^{\prime}$ (see the examples in Sec. IV).

Substituting Eq. (43) into Eq. (33), and then substituting the result into Eq. (24), we obtain

$$
Y_{p}\left(z^{\prime}, t\right) \sim \frac{G\left(z^{\prime}\right)}{\omega_{\beta}^{3} \sqrt{2 \pi}} \Phi\left(z^{\prime}\right) e^{-\Delta_{p}\left(t-z^{\prime} / v\right)} e^{-\Delta_{e} z^{\prime} / v} \int_{-\infty}^{\infty} \exp \left[i \omega\left(t-z^{\prime} / v\right)+\frac{i \omega_{\beta}^{2} J\left(z^{\prime}\right)}{\omega^{2}-\omega_{\beta}^{2}}\right] d \omega .
$$

The inverse Fourier transformation in Eq. (45) can be performed by using the result from Appendix B to obtain

$$
\begin{aligned}
Y_{p}\left(z^{\prime}, t\right) \sim & \sqrt{2 \pi} J\left(z^{\prime}\right) \xi\left(z^{\prime}\right) \Phi\left(z^{\prime}\right) \exp \left[-\Delta_{p}\left(t-\frac{z^{\prime}}{v}\right)-\Delta_{e}\left(\frac{z^{\prime}}{v}\right)-\frac{i J\left(z^{\prime}\right)}{4}\right] \\
& \times\left\{e^{-i \omega_{\beta}\left(t-z^{\prime} / v\right)}\left[\frac{I_{1}(u)}{u}-\frac{J^{2}\left(z^{\prime}\right) I_{2}(u)}{8 u^{2}}\right]-e^{i \omega_{\beta}\left(t-z^{\prime} / v\right)}\left[\frac{J_{1}(u)}{u}-\frac{J^{2}\left(z^{\prime}\right) J_{2}(u)}{8 u^{2}}\right]\right\},
\end{aligned}
$$

where $\xi\left(z^{\prime}\right)$ is defined in Eq. (16), $u$ is defined by

$$
u=u\left(z^{\prime}, t\right)=\sqrt{2 \omega_{\beta} J\left(z^{\prime}\right)\left(t-z^{\prime} / v\right)}
$$

$J_{n}(x)$ and $I_{n}(x)$ are the Bessel function of the first kind and the modified Bessel function of the first kind, respectively. Also, we have neglected the terms of higher order in $J\left(z^{\prime}\right) / u$ to derive Eq. (46). Using this approximate solution as a guide, we can obtain an exact solution of Eq. (32) in terms of an infinite series as discussed in Appendix A. The solution given in Eq. (46) is the same as that results from retaining the $n=0, m=1$ and 2 terms in the exact solution given in Eq. (A13) in Appendix A. Keeping more terms in the infinite series of the exact solution provides better accuracy, but complicates the subsequent discussion. Therefore we limit our exploration of the $e$ - $p$ instability to the solution given in Eq. (46).

We can derive a second approximate solution for $Y_{p}\left(z^{\prime}, \omega\right)$ by taking the complex conjugate of Eq. (46), or by making the substitution

$$
\hat{Y}\left(z^{\prime}, \omega\right)=\omega_{\beta}^{-3} \zeta\left(z^{\prime}, \omega\right) \Psi\left(z^{\prime}\right)
$$

in Eq. (36) and following the same procedures used in deriving Eq. (46). Either approach leads to the result

$$
\begin{aligned}
Y_{p}\left(z^{\prime}, t\right) \sim & \sqrt{2 \pi} J\left(z^{\prime}\right) \xi\left(z^{\prime}\right) \Psi\left(z^{\prime}\right) \exp \left[-\Delta_{p}\left(t-\frac{z^{\prime}}{v}\right)-\Delta_{e}\left(\frac{z^{\prime}}{v}\right)+\frac{i J\left(z^{\prime}\right)}{4}\right] \\
& \times\left\{e^{i \omega_{\beta}\left(t-z^{\prime} / v\right)}\left[\frac{I_{1}(u)}{u}-\frac{J^{2}\left(z^{\prime}\right) I_{2}(u)}{8 u^{2}}\right]-e^{-i \omega_{\beta}\left(t-z^{\prime} / v\right)}\left[\frac{J_{1}(u)}{u}-\frac{J^{2}\left(z^{\prime}\right) J_{2}(u)}{8 u^{2}}\right]\right\} .
\end{aligned}
$$

In terms of $R(x)$ and $\Theta(x)$ defined in Eqs. (39) and (40), the general solution for $Y_{p}\left(z^{\prime}, t\right)$ can be expressed as a linear combination of the solutions in Eqs. (46) and (49) by

$$
Y_{p}\left(z^{\prime}, t\right) \approx C_{p} M_{p}\left(z^{\prime}\right) e^{-\Delta_{p} t}\left\{\left[\frac{I_{1}(u)}{u}-\frac{J^{2}\left(z^{\prime}\right) I_{2}(u)}{8 u^{2}}\right] \cos T_{p}-\left[\frac{J_{1}(u)}{u}-\frac{J^{2}\left(z^{\prime}\right) J_{2}(u)}{8 u^{2}}\right] \cos S_{p}\right\},
$$

where

$$
\begin{gathered}
M_{p}\left(z^{\prime}\right)=J\left(z^{\prime}\right) \xi\left(z^{\prime}\right) R\left(z^{\prime}\right) \exp \left[\left(\Delta_{p}-\Delta_{e}\right) z^{\prime} / v\right], \\
T_{p}=T_{p}\left(z^{\prime}, t\right)=\sigma_{p}-\omega_{\beta}\left(t-z^{\prime} / v\right)+\Theta\left(z^{\prime}\right)-J\left(z^{\prime}\right) / 4,
\end{gathered}
$$

and

$$
S_{p}=S_{p}\left(z^{\prime}, t\right)=\sigma_{p}+\omega_{\beta}\left(t-z^{\prime} / v\right)+\Theta\left(z^{\prime}\right)-J\left(z^{\prime}\right) / 4
$$

Here, $C_{p}$ and $\sigma_{p}$ are constants. Since $\Theta\left(z^{\prime}\right)$ is the total phase advance of the $e-p$ oscillation, if we introduce the effective wave number $\hat{k} \approx \Theta\left(z^{\prime}\right) / z^{\prime}$ we recognize that $\cos \left[T_{p}\left(z^{\prime}, t\right)\right]$ represents a slow wave traveling in the positive $z^{\prime}$ direction, i.e., in the direction opposite to the proton beam's propagation. By the same reasoning, we recognize that $\cos \left[S\left(z^{\prime}, t\right)\right]$ represents a fast wave. Because the modified Bessel function $I_{n}(x)$ is a monotonically increasing function of $x$, Eq. (50) indicates that the slow wave is the one that grows - a well-known result [25].

The transformation from the beam-frame solution to the laboratory-frame solution can be easily performed by the substitution $z^{\prime}=z+v t$. We use Eq. (46) as an example. Thus, making the substitution $z^{\prime}=z+v t$ in Eq. (46) yields 


$$
\begin{aligned}
Y_{p}(z, t) \sim & \sqrt{2 \pi} J(z+v t) \xi(z+v t) \Phi(z+v t) \exp \left[-\Delta_{p}\left(\frac{z}{v}\right)-\Delta_{e}\left(t+\frac{z}{v}\right)-\frac{i J(z+v t)}{4}\right] \\
& \times\left\{e^{-i \omega_{\beta} z / v}\left[\frac{I_{1}(\tilde{u})}{\tilde{u}}-\frac{J^{2}(z+v t) I_{2}(\tilde{u})}{8 \tilde{u}^{2}}\right]-e^{i \omega_{\beta} z / v}\left[\frac{J_{1}(\tilde{u})}{\tilde{u}}-\frac{J^{2}(z+v t) J_{2}(\tilde{u})}{8 \tilde{u}^{2}}\right]\right\},
\end{aligned}
$$

where $\tilde{u}=\tilde{u}(z, t)=\sqrt{-2 \omega_{\beta} J(z+v t) z}, z \leq 0$, and $0 \leq v t+z \leq L$.

The Bessel function $J_{n}(u)$ goes to zero as $t$ increases. Therefore, if we are interested primarily in the growth of the $e-p$ motion, we can drop the terms containing $J_{n}(x)$ in Eq. (50) and obtain the approximate solution

$$
Y_{p}\left(z^{\prime}, t\right) \approx C_{p} M_{p}\left(z^{\prime}\right) e^{-\Delta_{p} t}\left[\frac{I_{1}(u)}{u}-\frac{J^{2}\left(z^{\prime}\right) I_{2}(u)}{8 u^{2}}\right] \cos T_{p}
$$

For large $t$, we can further neglect the term containing the Bessel function $I_{2}(u)$ and use the large-argument expansion $I_{n}(x) \approx e^{x} / \sqrt{2 \pi x}$ in Eq. (55) to yield the asymptotic solution of Eqs. (20) and (21); this gives

$$
\begin{aligned}
Y_{p}\left(z^{\prime}, t\right) \approx & \frac{C_{p}^{\prime} J\left(z^{\prime}\right) \xi\left(z^{\prime}\right) R\left(z^{\prime}\right)}{\left[2 \omega_{\beta} J\left(z^{\prime}\right)\left(t-z^{\prime} / v\right)\right]^{3 / 4}} \cos \left[\sigma+\Theta\left(z^{\prime}\right)-\omega_{\beta}\left(t-z^{\prime} / v\right)-J\left(z^{\prime}\right) / 4\right] \\
& \times \exp \left[-\Delta_{p}\left(t-\frac{z^{\prime}}{v}\right)-\Delta_{e}\left(\frac{z^{\prime}}{v}\right)+\sqrt{2 \omega_{\beta} J\left(z^{\prime}\right)\left(t-z^{\prime} / v\right)}\right]
\end{aligned}
$$

where $C_{p}^{\prime}$ is a constant.

The transverse motion of the electron centroid within the extent of the proton bunch is treated in Appendix A. By retaining the $n=0, m=2$, and $m=3$ terms in Eq. (A17), we obtain an approximate solution for $Y_{e}\left(z^{\prime}, t\right)$ to lowest order in $J\left(z^{\prime}\right) / u$. We find

$$
\begin{aligned}
Y_{e}\left(z^{\prime}, t\right) \sim & -i 2 \sqrt{2 \pi} J^{2}\left(z^{\prime}\right) \Phi\left(z^{\prime}\right) \exp \left[-\frac{i J\left(z^{\prime}\right)}{4}-\Delta_{p}\left(t-\frac{z^{\prime}}{v}\right)-\Delta_{e}\left(\frac{z^{\prime}}{v}\right)\right] \\
& \times\left\{e^{-i \omega_{\beta}\left(t-z^{\prime} / v\right)}\left[\frac{I_{2}(u)}{u^{2}}+\frac{i J\left(z^{\prime}\right) I_{3}(u)}{2 u^{3}}\right]-e^{i \omega_{\beta}\left(t-z^{\prime} / v\right)}\left[\frac{J_{2}(u)}{u^{2}}-\frac{i J\left(z^{\prime}\right) J_{3}(u)}{2 u^{3}}\right]\right\} .
\end{aligned}
$$

By including the solution containing $\Psi\left(z^{\prime}\right)$, we can derive the growing part of $Y_{e}\left(z^{\prime}, t\right)$. This gives

$$
Y_{e}\left(z^{\prime}, t\right) \approx C_{e} M_{e}\left(z^{\prime}\right) e^{-\Delta_{p} t}\left[\frac{I_{2}^{2}(u)}{u^{4}}+\frac{J^{2}\left(z^{\prime}\right) I_{3}^{2}(u)}{4 u^{6}}\right]^{1 / 2} \cos T_{e},
$$

where

$$
\begin{gathered}
M_{e}\left(z^{\prime}\right)=J^{2}\left(z^{\prime}\right) R\left(z^{\prime}\right) \exp \left[\left(\Delta_{p}-\Delta_{e}\right) z^{\prime} / v\right], \\
T_{e}=T_{e}\left(z^{\prime}, t\right)=\psi_{e}+\sigma_{e}-\omega_{\beta}\left(t-z^{\prime} / v\right)+\Theta\left(z^{\prime}\right)-J\left(z^{\prime}\right) / 4, \\
\psi_{e}=\cos ^{-1}\left\{u I_{2}(u) /\left[4 u^{2} I_{2}^{2}(u)+J^{2}\left(z^{\prime}\right) I_{3}^{2}(u)\right]^{1 / 2}\right\},
\end{gathered}
$$

and $C_{e}$ and $\sigma_{e}$ are constants. The asymptotic solution for $Y_{e}$ and the ratio $\left|Y_{e} / Y_{p}\right|$ at large $t$ can be derived in the same way as Eq. (56). Carrying out the necessary algebra, we obtain

$$
\begin{aligned}
Y_{e}\left(z^{\prime}, t\right) \approx & \frac{C_{e}^{\prime} J^{2}\left(z^{\prime}\right) R\left(z^{\prime}\right)}{\left[2 \omega_{\beta} J\left(z^{\prime}\right)\left(t-z^{\prime} / v\right)\right]^{5 / 4}} \cos \left[\psi_{e}+\sigma_{e}+\Theta\left(z^{\prime}\right)-\omega_{\beta}\left(t-z^{\prime} / v\right)-J\left(z^{\prime}\right) / 4\right] \\
& \times \exp \left[-\Delta_{p}\left(t-\frac{z^{\prime}}{v}\right)-\Delta_{e}\left(\frac{z^{\prime}}{v}\right)+\sqrt{2 \omega_{\beta} J\left(z^{\prime}\right)\left(t-z^{\prime} / v\right)}\right],
\end{aligned}
$$

and

$$
\left|\frac{Y_{e}\left(z^{\prime}, t\right)}{Y_{p}\left(z^{\prime}, t\right)}\right| \propto \frac{1}{\xi\left(z^{\prime}\right)} \sqrt{\frac{J\left(z^{\prime}\right)}{2 \omega_{\beta}\left(t-z^{\prime} / v\right)}},
$$


where $C_{e}^{\prime}$ is a constant. Note that neither $\Delta_{p}$ nor $\Delta_{e}$ appears in Eq. (63). In fact, using the exact solutions, Eqs. (A12) and (A16) in Appendix A, we can show that the ratio $Y_{e} / Y_{p}$ is always independent of the damping due to frequency spreads.

\section{B. Growth rate and dispersion relation}

The results derived in Secs. II and III are applicable to a large class of proton and electron line densities. From these general solutions, we note that how the unstable mode "wiggles" in space is determined primarily by the factor $\Phi \mathrm{e}^{-i J\left(z^{\prime}\right) / 4}$, where $\Phi\left(z^{\prime}\right)$ is the "cardiograph" of the electron vibration in the proton bunch, and the quantity $-i J\left(z^{\prime}\right) / 4$ represents the accumulated phase shift in the proton centroid's wiggle from the head of the bunch to the location $z^{\prime}$ due to the interaction between the protons and the electron cloud. We also note that $J\left(z^{\prime}\right)$ plays the major role in determining the spatial growth [growth along the proton bunch like $\exp (\alpha \sqrt{J})]$ of the instability. As indicated in Eqs. (56) and (62), at large $t$, the instability always grows like $\exp (\alpha \sqrt{t})$ in the beam frame. For a Lorentzian frequency spread, the $e$ - $p$ mode damps in time like $\exp \left(-\Delta_{p} t\right)$. We further note that due to the combination of $\exp (\alpha \sqrt{t})$ growth and $\exp \left(-\Delta_{p} t\right)$ damping, a perturbation can grow initially and eventually be damped even for a very small nonzero value of $\Delta_{p}$. This important feature originates from combining the one-pass twostream interaction and the Lorentzian frequency spread in the present model.

We define the growth or damping rate, $\Gamma_{q}\left(z^{\prime}, t\right)$, as a function of $z^{\prime}$ and $t$ according to

$$
\Gamma_{q}\left(z^{\prime}, t\right)=\frac{1}{\left[Y_{q}\left(z^{\prime}, t\right)\right]_{a}} \frac{\partial\left[Y_{q}\left(z^{\prime}, t\right)\right]_{a}}{\partial t},
$$

where the subscript $q$ stands for either protons $(p)$, or electrons $(e)$, and $\left[Y_{q}\left(z^{\prime}, t\right)\right]_{a}$ denotes the amplitude of the oscillation described by $Y_{q}\left(z^{\prime}, t\right)$. Substituting Eqs. (55) and (58) into Eq. (64) yields the following growth rates of the $e-p$ mode given in Eqs. (46) and (57):

$$
\Gamma_{p}\left(z^{\prime}, t\right) \approx-\Delta_{p}+\frac{\omega_{\beta} J\left(z^{\prime}\right)\left[8 u I_{2}(u)-J^{2}\left(z^{\prime}\right) I_{3}(u)\right]}{u\left[8 u I_{1}(u)-J^{2}\left(z^{\prime}\right) I_{2}(u)\right]},
$$

and

$$
\begin{aligned}
\Gamma_{e}\left(z^{\prime}, t\right) \approx & -\Delta_{p} \\
& +\frac{\omega_{\beta} J\left(z^{\prime}\right) I_{3}(u)\left[4 u^{2} I_{2}(u)+J^{2}\left(z^{\prime}\right) I_{4}(u)\right]}{u\left[4 u^{2} I_{2}^{2}(u)+J^{2}\left(z^{\prime}\right) I_{3}^{2}(u)\right]},
\end{aligned}
$$

where $u=u\left(z^{\prime}, t\right)=\sqrt{2 \omega_{\beta} J\left(z^{\prime}\right)\left(t-z^{\prime} / v\right)}$. For $u \gg 1$, we obtain

$$
\Gamma_{q}\left(z^{\prime}, t\right) \approx-\Delta_{p}+\sqrt{\frac{\omega_{\beta} J\left(z^{\prime}\right)}{2\left(t-z^{\prime} / v\right)}} .
$$

Thus, the instability damps after $t \approx\left(z^{\prime} / v\right)+$ $\omega_{\beta} J\left(z^{\prime}\right) /\left(2 \Delta_{p}^{2}\right)$. Since $J\left(z^{\prime}\right)$ is typically less than 1 for small fractional charge neutralizations (like a few percent), the growth rates in Eqs. (65) and (66) are monotonically decreasing functions of time with maxima located at $t=z^{\prime} / v$. Hence the threshold at location $z^{\prime}$, $\left(\Delta_{p} / \omega_{\beta}\right)_{t}$, can be found by solving the equation $\Gamma_{q}\left(z^{\prime}, t=\right.$ $\left.z^{\prime} / v\right)=0$. This yields

$$
\left(\frac{\Delta_{p}}{\omega_{\beta}}\right)_{t} \approx \max \left\{\frac{J\left(z^{\prime}\right)}{4}\left[\frac{1-J^{2}\left(z^{\prime}\right) / 48}{1-J^{2}\left(z^{\prime}\right) / 32}\right]\right\},
$$

where $\max \left\{f\left(z^{\prime}\right)\right\}$ indicates the maximum of $f\left(z^{\prime}\right)$ for $0 \leq$ $z^{\prime} \leq L$. Because $J\left(z^{\prime}\right)$ is a monotonically increasing function of $z^{\prime}$, the largest growth rate and the lowest threshold are at the tail of the proton bunch. Equation (68) is valid only for small $J\left(z^{\prime}\right)$, otherwise more terms in the series of the exact solution should be included.

One should keep in mind that when $t$ is small, Eqs. (65) and (66) are applicable only to the $e-p$ mode described in Eqs. (46) and (57). Likewise, the validity of Eq. (68) is strictly limited to the $e-p$ mode. Initial perturbations different from those described in Eqs. (46) and (57), e.g., pure sine waves, etc., can have quite different thresholds and will take a different time interval to evolve into the $e-p$ mode. Therefore, applying Eqs. (65), (66), and (68) blindly may result in incorrect conclusions. Nonetheless, after the initial perturbation has evolved into the $e-p$ mode, the asymptotic growth rate given in Eq. (67) tends to provide a good approximation.

From Eqs. (46)-(57), we see that the electron frequency spread always causes spatial damping in the positive $z^{\prime}$ direction (i.e., more damping in the tail of the proton bunch since the protons here are traveling in the negative $z^{\prime}$ direction), but it does not damp the perturbation in time. On the other hand, the proton frequency spread not only damps the perturbation in time but also introduces a "damping offset" (the $e^{\Delta_{p} z^{\prime} / v}$ factor) which looks like antidamping so that there is an apparent competition between the spatial damping and the fallacious antidamping due to these two frequency spreads. When the frequency spread of protons is equal to that of electrons, the associated spatial damping and damping offset cancel each other exactly. The origin of this spurious antidamping or the damping offset can be delineated through a simple physical picture. Suppose both the protons and the electrons were in equilibrium before $t=0$, i.e., $d Y_{p} / d t=d Y_{e} / d t=Y_{p}=Y_{e}=0$ for $t<0$. At $t=0$, a delta-function-like electron perturbation enters the proton bunch from the head. As soon as the head starts to move, the damping of the motion at the head also begins, while the rest of the bunch remains quiescent (not 
perturbed). This perturbation pulse takes a time interval of $t=z^{\prime} / v$ to travel to the point $z^{\prime}$ in the bunch to initiate the proton motion and the damping there. Hence the damping at $z^{\prime}$ should follow $\exp \left[-\Delta_{p}\left(t-z^{\prime} / v\right)\right]$ or $\exp \left(-\Delta_{p} t\right) \exp \left(\Delta_{p} z^{\prime} / v\right)$. Thus the damping offset $\exp \left(\Delta_{p} z^{\prime} / v\right)$ is caused by the assumption that the part of the proton bunch before the point $z^{\prime}$ is perturbed and by the electrons' time of flight between the head and the location $z^{\prime}$ in the proton bunch. When $\Delta_{p}=\Delta_{e}$, the alltime cancellation between the spatial damping due to the electron frequency spread and the damping offset is possible only when the electrons and the protons have the same frequency distributions, i.e., both are Lorentzian or both are Gaussian.

The discussion so far has concentrated on the timedomain solution which allows us to understand some behavior of the system directly. In principle, the same physics can be revealed in a frequency-domain analysis. However, because the charge densities of the protons and electrons can be highly nonuniform in bunched beams, the function $e^{i(\omega t-k z)}$ customarily used in studying perturbations about uniform plasmas is not a solution in the bunched beam case. Thus, in the frequency domain, physical results are obtained in a less direct way by superposing globally uniform modes of constant growth or damping rates. On the other hand, knowing the frequency response of the $e-p$ system does have several advantages, such as predicting the system behavior when it interacts with an environment of known frequency characteristics (machine impedance), or in helping to mitigate the instability (e.g., feedback damping). Therefore, although the emphasis of this work is on the time-domain study, we derive here a dispersion relation in the frequency domain. For simplicity, we consider the special case of a uniform electron background, i.e., $G(z)$ is a constant, in the following analysis.

Making Fourier transformations of Eqs. (20) and (21) from the time $(t)$ domain to the frequency $(\omega)$ domain, and eliminating the Fourier transform of $Y_{e}\left(z^{\prime}, t\right)$ from the resulting equations, we obtain

$$
\frac{d^{2} \tilde{Y}_{p}}{d z^{\prime 2}}+2\left(\frac{\Delta_{e}+i \omega}{v}\right) \frac{d \tilde{Y}_{p}}{d z^{\prime}}+\left[\left(\frac{\Delta_{e}+i \omega}{v}\right)^{2}+\frac{\Omega^{2}\left(z^{\prime}\right)}{v^{2}}\left(1-\frac{G}{g}\right)\right] \tilde{Y}_{p}=0
$$

where

$$
g=\omega_{\beta}^{2}-\left(\omega-i \Delta_{p}\right)^{2},
$$

and $\tilde{Y}_{p}\left(z^{\prime}, \omega\right)$ is the Fourier transform of $Y_{p}\left(z^{\prime}, t\right)$. Performing a Fourier transformation of Eq. (69) according to

$$
\int_{-\infty}^{\infty}\left\{\frac{d^{2} \tilde{Y}_{p}}{d z^{\prime 2}}+2\left(\frac{\Delta_{e}+i \omega}{v}\right) \frac{d \tilde{Y}_{p}}{d z^{\prime}}+\left[\left(\frac{\Delta_{e}+i \omega}{v}\right)^{2}+\frac{\Omega^{2}\left(z^{\prime}\right)}{v^{2}}\left(1-\frac{G}{g}\right)\right] \tilde{Y}_{p}\right\} e^{i k z^{\prime}} d z^{\prime}=0,
$$

yields

$$
\left(k v-\omega+i \Delta_{e}\right)^{2} \int_{-\infty}^{\infty} \tilde{Y}_{p}\left(z^{\prime}, \omega\right) e^{i k z^{\prime}} d z^{\prime}=\left(1-\frac{G}{g}\right) \int_{-\infty}^{\infty} \Omega^{2}\left(z^{\prime}\right) \tilde{Y}_{p}\left(z^{\prime}, \omega\right) e^{i k z^{\prime}} d z^{\prime} .
$$

Then, from Eqs. (24), (33), (35), (37), and (42), we infer that

$$
\tilde{Y}_{p}\left(z^{\prime}, \omega\right) \approx G \hat{Y}\left(z^{\prime}, \omega-i \Delta_{p}\right) e^{-i\left(\omega-i \Delta_{e}\right) z^{\prime} / v}=G \omega_{\beta}^{-3} \Phi\left(z^{\prime}\right) \exp \left[-i\left(\omega-i \Delta_{e}\right)\left(\frac{z^{\prime}}{v}\right)+\frac{i \omega_{\beta}^{2} J\left(z^{\prime}\right)}{\left(\omega-i \Delta_{p}\right)^{2}-\omega_{\beta}^{2}}\right],
$$

where $\hat{Y}\left(z^{\prime}, \omega\right)$ is defined in Eq. (37). Substituting Eq. (73) into Eq. (72) yields the following dispersion relation in the beam frame:

$$
\left(\omega-i \Delta_{e}-k v\right)^{2}\left[\omega_{\beta}^{2}-\left(\omega-i \Delta_{p}\right)^{2}\right] \approx\left[\omega_{\beta}^{2}-\left(\omega-i \Delta_{p}\right)^{2}-G\right] \Lambda(k, \omega)
$$

where

$$
\Lambda(k, \omega)=\frac{\int_{-\infty}^{\infty} \Omega^{2}\left(z^{\prime}\right) \Phi\left(z^{\prime}\right) \exp \left[i k z^{\prime}-i\left(\omega-i \Delta_{e}\right)\left(\frac{z^{\prime}}{v}\right)+\frac{i \omega_{\beta}^{2} J\left(z^{\prime}\right)}{\left(\omega-i \Delta_{p}\right)^{2}-\omega_{\beta}^{2}}\right] d z^{\prime}}{\int_{-\infty}^{\infty} \Phi\left(z^{\prime}\right) \exp \left[i k z^{\prime}-i\left(\omega-i \Delta_{e}\right)\left(\frac{z^{\prime}}{v}\right)+\frac{i \omega_{\beta}^{2} J\left(z^{\prime}\right)}{\left(\omega-i \Delta_{p}\right)^{2}-\omega_{\beta}^{2}}\right] d z^{\prime}} .
$$

To rewrite the dispersion relation in laboratory-frame notation, we simply replace $\omega$ in Eq. (74) by $\omega+k v$. In addition, we may also make the substitution $k=n \Omega_{r} / v$ for circular machine applications, where $\Omega_{r}$ is the angular revolution frequency of the protons in the machine. For the case of a proton bunch propagating in the positive $z$ direction, the sign of $v$ should be reversed. Equation (74) has been derived for constant $G\left(z^{\prime}, t\right)$. For a nonuniform, time-independent electron line density, $d G / d z^{\prime}$ appears 
in the coefficients of Eq. (69), and the dispersion relation is more complex than Eq. (74).

\section{EXAMPLES}

We now present two examples of specific line density profiles to illustrate the application of the theory developed here. Numerical results will be presented for the case of a parabolic proton line density and a uniform electron line density.

\section{A. Uniform proton and electron line densities}

When both the proton and the electron line densities are uniform in $z^{\prime}$, we can infer that the fractional charge neutralization $f$ is a constant, $\Omega^{2}\left(z^{\prime}\right)=\omega_{o}^{2}, R\left(z^{\prime}\right)=1$, $\Theta\left(z^{\prime}\right)=\omega_{o} z^{\prime} / v$, and $G\left(z^{\prime}\right)=\xi \omega_{\beta}^{2}$, where $\omega_{o}=$ $(c / a) \sqrt{2 r_{e} N(1-f) / L}$ is the electron bounce frequency in a proton bunch containing $N$ protons, and $\xi$ is a constant. Substituting into Eqs. (44) and (46), we obtain

$$
J\left(z^{\prime}\right)=\frac{\xi \omega_{o} z^{\prime}}{2 v}=\frac{r_{p} c^{2} \lambda_{e} \omega_{o} z^{\prime}}{a^{2} \gamma \omega_{\beta}^{2} v}
$$

$$
Y_{p}\left(z^{\prime}, t\right) \approx C z^{\prime} e^{-\Delta_{p} t+\left(\Delta_{p}-\Delta_{e}\right) z^{\prime} / v}\left\{\left[\frac{I_{1}(u)}{u}-\frac{J^{2}\left(z^{\prime}\right) I_{2}(u)}{8 u^{2}}\right] \cos T-\left[\frac{J_{1}(u)}{u}-\frac{J^{2}\left(z^{\prime}\right) J_{2}(u)}{8 u^{2}}\right] \cos S\right\},
$$

where $T=\sigma+\left(\omega_{o}+\omega_{\beta}-\xi \omega_{o} / 8\right)\left(z^{\prime} / v\right)-\omega_{\beta} t, S=\sigma+\left(\omega_{o}-\omega_{\beta}-\xi \omega_{o} / 8\right)\left(z^{\prime} / v\right)+\omega_{\beta} t, C$ and $\sigma$ are constants, and $u=\sqrt{\xi \omega_{o} \omega_{\beta}\left(z^{\prime} / v\right)\left(t-z^{\prime} / v\right)}$. At large $t$, Eq. (77) gives

$$
\begin{aligned}
Y_{p}\left(z^{\prime}, t\right) \approx & \hat{C}\left[\frac{\omega_{o} z^{\prime}}{v \omega_{\beta}^{3}\left(t-z^{\prime} / v\right)^{3}}\right]^{1 / 4} \cos \left[\sigma+\left(\omega_{o}+\omega_{\beta}-\xi \omega_{o} / 8\right)\left(z^{\prime} / v\right)-\omega_{\beta} t\right] \\
& \times \exp \left[-\Delta_{p}\left(t-\frac{z^{\prime}}{v}\right)-\Delta_{e}\left(\frac{z^{\prime}}{v}\right)+\sqrt{\xi \omega_{o} \omega_{\beta}\left(\frac{z^{\prime}}{v}\right)\left(t-\frac{z^{\prime}}{v}\right)}\right]
\end{aligned}
$$

where $\hat{C}$ is a constant. The asymptotic growth rate of the $e-p$ mode for this case can be determined by substituting the result for $J\left(z^{\prime}\right)$ in Eq. (76) into Eq. (67). Thus, for large $t$, we obtain

$$
\Gamma\left(z^{\prime}, t\right) \approx-\Delta_{p}+\sqrt{\frac{\xi \omega_{o} \omega_{\beta} z^{\prime}}{4\left(v t-z^{\prime}\right)}} .
$$

When both the protons and the electrons have uniform line densities, $\Lambda(k, \omega)=\omega_{o}^{2}$, and the dispersion relation, Eq. (74), simplifies to give

$$
\left[\left(\omega-i \Delta_{e}-k v\right)^{2}-\omega_{o}^{2}\right]\left[\left(\omega-i \Delta_{p}\right)^{2}-\omega_{\beta}^{2}\right]=G \omega_{o}^{2}
$$

This result is similar to the dispersion relations derived in some earlier works[12,13,19-25]. As stated earlier, for a circular machine with proton angular revolution frequency $\Omega_{r}$, one can make the substitution $k=n \Omega_{r} / v$ in Eq. (80).

For nonuniform proton line densities, there are a few cases in which Eq. (28) has well-known solutions. Examples are the parabolic cylinder function and the Mathieu function solutions for parabolic and cosinesquare proton line densities, respectively. The following example demonstrates the application of Eqs. (46) -(62) to the case of parabolic proton line density. For this purpose, it is sufficient to determine the solutions $\Phi(x)$ and $\Psi(x)$, and to evaluate $J(x)$. The solutions for $Y_{p}\left(z^{\prime}, t\right)$ and
$Y_{e}\left(z^{\prime}, t\right)$ can then be obtained by substituting these results into Eqs. (50) and (57), respectively.

\section{B. Parabolic proton line density and uniform electron line density}

We consider the proton line density

$$
\lambda_{p}\left(z^{\prime}\right)=\frac{3 N}{\left(2+f_{o}\right) L}\left[f_{o}+4\left(1-f_{o}\right) \frac{z^{\prime}}{L}\left(1-\frac{z^{\prime}}{L}\right)\right]
$$

and the electron line density

$$
\lambda_{e}\left(z^{\prime}\right)=\lambda_{e}=\frac{3 N f_{o}}{\left(2+f_{o}\right) L},
$$

where $N$ is the number of protons in the bunch, and $f_{o}$ is the fractional charge neutralization at the center of the proton bunch. In fact, the charge of $\lambda_{p}\left(z^{\prime}\right)$ in Eq. (81) is a superposition of parabolic and uniform line densities with $\lambda_{p}(0)=\lambda_{p}(L)=\lambda_{e}$. For small (zero) fractional neutralization, $\lambda_{p}$ is almost (exactly) parabolic. The system has a net charge line density that is parabolic.

In this case, $\xi(x)$ and $G(x)$ are constants, and the electron bounce frequency is given by

$$
\Omega^{2}\left(z^{\prime}\right)=\frac{4 \omega_{o}^{2} z^{\prime}}{L}\left(1-\frac{z^{\prime}}{L}\right),
$$

where $\omega_{o}=(c / a) \sqrt{6 r_{e} N\left(1-f_{o}\right) /\left[\left(2+f_{o}\right) L\right]}$ is the electron bounce frequency at the center of the proton bunch. The functions $\Phi(x)$ and $\Psi(x)$ will be determined by 
solving Eq. (28). Rewriting $\Omega^{2}\left(z^{\prime}\right)$ in laboratory-frame variables and substituting into Eq. (28) yields

$$
\frac{d^{2} Y_{e n}}{d t^{2}}+\frac{\omega_{o}^{2} v}{L}\left(t+\frac{z}{v}\right)\left[1-\frac{v}{L}\left(t+\frac{z}{v}\right)\right] Y_{e n}=0 .
$$

We change variables by introducing

$$
h=\frac{L \omega_{o}}{4 v},
$$

and

$$
p=2 \sqrt{\frac{\omega_{o}}{L}}\left(\frac{z^{\prime}}{v}-\frac{L}{2 v}\right)=2 \sqrt{\frac{\omega_{o} v}{L}}\left(t+\frac{z}{v}-\frac{L}{2 v}\right) .
$$

Equation (84) can then be rewritten as

$$
\frac{d^{2} Y_{e n}}{d p^{2}}+\left(h-\frac{p^{2}}{4}\right) Y_{e n}=0 .
$$

The two independent solutions to Eq. (87), known as the parabolic cylinder functions, are given by [33]

$$
U(-h, p)=Y_{1} \cos \left[\frac{\pi}{2}\left(\frac{1}{2}-h\right)\right]-Y_{2} \sin \left[\frac{\pi}{2}\left(\frac{1}{2}-h\right)\right],
$$

and

$$
\begin{aligned}
V(-h, p)=\frac{1}{\Gamma\left(\frac{1}{2}+h\right)}\{ & Y_{1} \sin \left[\frac{\pi}{2}\left(\frac{1}{2}-h\right)\right] \\
& \left.+Y_{2} \cos \left[\frac{\pi}{2}\left(\frac{1}{2}-h\right)\right]\right\},
\end{aligned}
$$

where

$$
Y_{1}=\frac{\Gamma\left(\frac{1}{4}+\frac{h}{2}\right)}{2^{(1 / 4)-(h / 2)} \sqrt{\pi}} e^{-p^{2} / 4}{ }_{1} F_{1}\left(\frac{1}{4}-\frac{h}{2 ;} \frac{1}{2} ; \frac{p^{2}}{2}\right),
$$

and

$$
Y_{2}=\frac{\Gamma\left(\frac{3}{4}+\frac{h}{2}\right)}{2^{-(1 / 4)-(h / 2)} \sqrt{\pi}} p e^{-p^{2} / 4}{ }_{1} F_{1}\left(\frac{3}{4}-\frac{h}{2} ; \frac{3}{2} ; \frac{p^{2}}{2}\right) .
$$

Here, $\Gamma(x)$ is the Gamma function, and ${ }_{1} F_{1}(a ; b ; x)$ is the confluent hypergeometric function [33]. The parabolic cylinder functions are similar to the Hermite polynomials $H_{n}(x)$. For $|p| \leq 2 \sqrt{h}$, or within the bunch length, both $U(a, x)$ and $V(a, x)$ oscillate like $e^{-x^{2} / 4} H_{n}(x)$, i.e., they have short wavelengths at small $x$ (near the bunch center where the proton density is high) and long wavelengths when $|x|$ (or $|p|$ ) approaches $2 \sqrt{h}$ (tails of the proton bunch where the line density is low). In fact, when $h=n+1 / 2, U(-h, p)$ becomes $2^{-n / 2} \mathrm{e}^{-p^{2} / 4} H_{n}(p / \sqrt{2})$. Hence there is an interesting analogy between Eq. (87) and the Schrödinger equation for a particle moving in a harmonic oscillator potential well. That is, if the bunch length $L$ is quantized to exactly $4 n+2$ times the electron bounce wavelength $v / \omega_{o}$, then the solution to Eq. (87) would be $e^{-p^{2} / 4} H_{n}(p / \sqrt{2})$.

In order to represent $\Phi\left(z^{\prime} / v\right)$ and $\Psi\left(z^{\prime} / v\right)$ in the polar forms shown in Eqs. (39) and (40), we use the linearly independent combinations of $U(a, x)$ and $V(a, x)$ defined by

$$
\begin{aligned}
\Phi\left(z^{\prime}\right)= & U(-h, p) \\
& +i \Gamma\left(\frac{1}{2}+h\right) V(-h, p)=R(h, p) e^{i \Theta(h, p)},
\end{aligned}
$$

and

$$
\begin{aligned}
\Psi\left(z^{\prime}\right)= & U(-h, p) \\
& -i \Gamma\left(\frac{1}{2}+h\right) V(-h, p)=R(h, p) e^{-i \Theta(h, p)} .
\end{aligned}
$$

Here, $R(h, p)$ and $\Theta(h, p)$ have the following expansions valid for $|p| \ll 2 \sqrt{h}[33]$ :

$$
\begin{aligned}
R(h, p) \approx & \frac{\Gamma\left(\frac{1}{4}+\frac{h}{2}\right)}{2^{(1 / 4)-(h / 2)} \sqrt{\pi}} \\
& \times\left[1+\left(\frac{p}{4 \sqrt{h}}\right)^{2}+\frac{5}{2}\left(\frac{p}{4 \sqrt{h}}\right)^{4}+\cdots\right],
\end{aligned}
$$

and

$$
\begin{aligned}
\Theta(h, p) \approx\left(\frac{1}{4}-\frac{h}{2}\right) \pi+\sqrt{h} p & {\left[1-\frac{2}{3}\left(\frac{p}{4 \sqrt{h}}\right)^{2}\right.} \\
& \left.-\frac{1}{(4 \sqrt{h})^{4}}\left(\frac{2}{5} p^{4}-16\right)+\cdots\right] .
\end{aligned}
$$

Equations (94) and (95) show that $R(h, p)$ and $d \Theta(h, p) / d p$ are smooth functions of $p$. Note that $R(h, p)$ and $d \Theta(h, p) / d p$ are symmetric with respect to $p$. Also note that the electron bounce frequency $\Theta(h, p)$ is not exactly proportional to the square root of the proton line density except at $z^{\prime}=L / 2$.

Using the Wronskian of $U$ and $V, W(U, V)=-\sqrt{2 / \pi}$, and Eq. (94), we find that

$$
W=\Psi \frac{d \Phi}{d x}-\Phi \frac{d \Psi}{d x}=4 i \sqrt{\frac{2 \omega_{o}}{\pi v L}} \Gamma\left(\frac{1}{2}+h\right) .
$$

Substituting Eqs. (83), (94), and (96) into Eq. (44) and carrying out the integration, we obtain

$J\left(z^{\prime}\right) \approx \frac{3 \xi \omega_{o} L}{2 v}\left(\frac{z^{\prime}}{L}\right)^{2}\left[1-\frac{14}{9}\left(\frac{z^{\prime}}{L}\right)+\frac{4}{3}\left(\frac{z^{\prime}}{L}\right)^{2}-\frac{8}{15}\left(\frac{z^{\prime}}{L}\right)^{3}\right]$,

where only the zeroth and the first-order terms in the expansion in $R^{2}$ have been retained. Thus, $J\left(z^{\prime}\right)$ is a monotonically increasing function of $z^{\prime}$, starting from zero at the head of the bunch and increasing to about $0.37 \xi \omega_{o} L / v$ at the tail of the bunch.

The solutions for $Y_{p}\left(z^{\prime}, t\right)$ and $Y_{e}\left(z^{\prime}, t\right)$ can be obtained by substituting Eqs. (92), (93), and (97) into 
Eqs. (50)-(53) and (57). One can also study the dispersion relation by making the substitution of $\Phi\left(z^{\prime}\right)=U(-h, p)$ or $\Phi\left(z^{\prime}\right)=V(-h, p)$ in Eq. (74). A comment should be given here on the use of the approximation in Eq. (94), which is valid only when $|p| \ll 2 \sqrt{h}$. Note that the expression for $J(x)$ contains the proton line density $\lambda_{p}(z / L)$ in the factor of $\Omega^{2}(z / L)$ in the integrand. Equation (94) is least accurate when $|p| \approx 2 \sqrt{h}$, or at the two ends of the bunch where $\Omega^{2}$ is small. Thus, the overall approximation should be reasonably good.

As a numerical example, we consider a set of parameter values similar to those in PSR: a 260 ns-long bunch of $2.54 \times 10^{13}, 800 \mathrm{MeV}$ protons circulating at a $2.8 \mathrm{MHz}$ revolution frequency in a storage ring operated at a betatron tune of 2.3. We assume that the tune spread of the protons is about $0.2 \%$, and that the proton bunch has a circular cross section with $2.5 \mathrm{~cm}$ radius. We also assume the fractional charge neutralization by the electrons at the bunch center is about $1 \%$, and that the oscillation frequency spread of the electrons is 5\%. For these parameter values, the electron bounce frequency at the bunch center is about $108.3 \mathrm{MHz}\left(\omega_{o} \approx 0.687 \mathrm{GHz}\right)$, and $\xi \approx 8.4 \times$ $10^{-4}$. Figure 1 shows the parabolic cylinder function solutions to Eq. (87), $U$ and $V$, which describe the transverse motion of an electron in the proton bunch. In the figure, the functions $U$ and $V$ are normalized to $R(h, 0) / \Gamma(1 / 2+h)$ and $R(h, 0)$, respectively. Shown in Fig. 2 is the quantity $J\left(z^{\prime}\right)$ plotted as a function of the distance from the head to the tail of the proton bunch, where both the approximation made by using Eq. (97) and the more exact values computed using Eq. (44) are displayed. The maximum of $J\left(z^{\prime}\right)$ is about 0.057 . According to Eq. (68), the instability threshold is near $\left(\Delta_{p} / \omega_{\beta}\right)_{t} \approx$ $1.4 \%$. So the case we are considering is theoretically

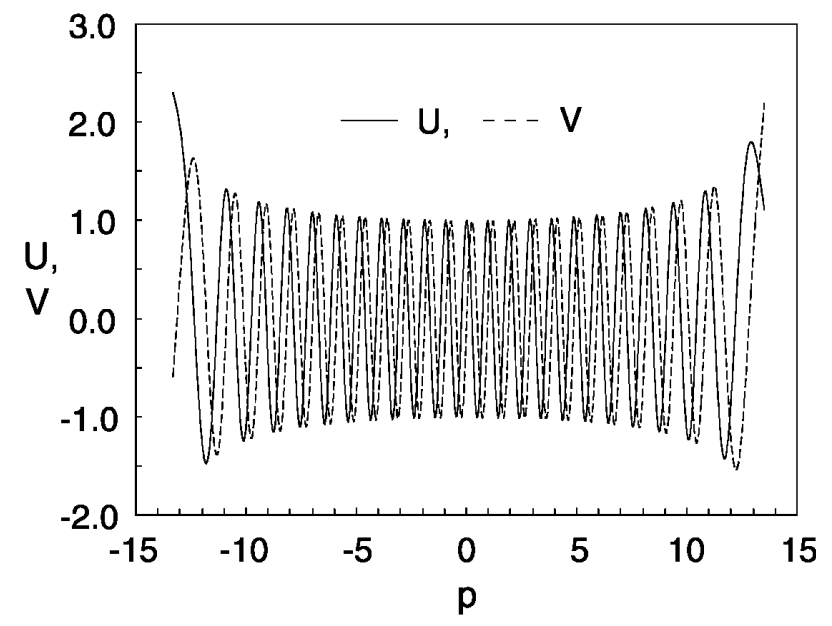

FIG. 1. The parabolic cylinder functions $U$ and $V$ as functions of $p$ defined in Eq. (86). Here, $U$ and $V$ are normalized to $R(h, 0)$ and $R(h, 0) / \Gamma(1 / 2+h)$, respectively; $h$ and $R(h, 0)$ are computed using Eqs. (85), (92), and (93) and the parameter values described in the text.

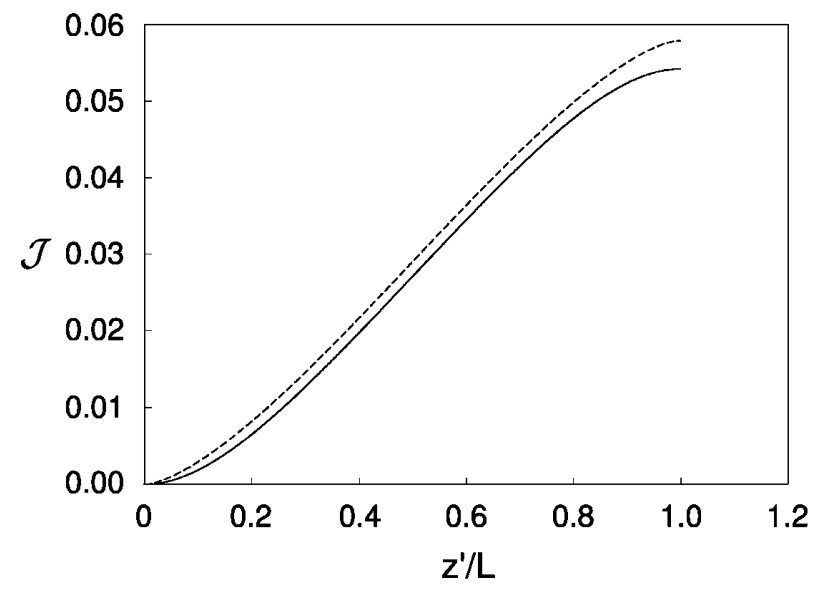

FIG. 2. The quantity $J$ as a function of $z^{\prime} / L$ from the head to the end of the proton bunch. The solid curve is computed using the approximation in Eq. (97). The dashed curve is computed by using Eq. (44) and numerical integration. The parameter values are described in the text.

unstable. The growth rates (normalized to the betatron frequency $\omega_{\beta}$ ) evaluated using Eqs. (65)-(67) for $z^{\prime}=$ $L / 2$ and $z^{\prime}=L$ are shown in Fig. 3 as functions of $z^{\prime} / v$. We see from Fig. 3 that the proton motion has a slightly higher growth rate than that of the electron motion. The observed instability threshold in PSR varies with machine conditions. For the parameter values we are considering, the instability typically occurs when $\left(\Delta_{p} / \omega_{\beta}\right)_{t}<0.5 \%$. Hence the threshold given by Eq. (68) overestimates the required frequency spread in protons for stabilization. Figure 4 shows the growing part of the perturbed proton motion $Y_{p}\left(z^{\prime}\right)$ computed using Eq. (55) for $\sigma_{p}=0$, and $t=40 \mu \mathrm{s}, 70 \mu \mathrm{s}$, and $100 \mu \mathrm{s}$.

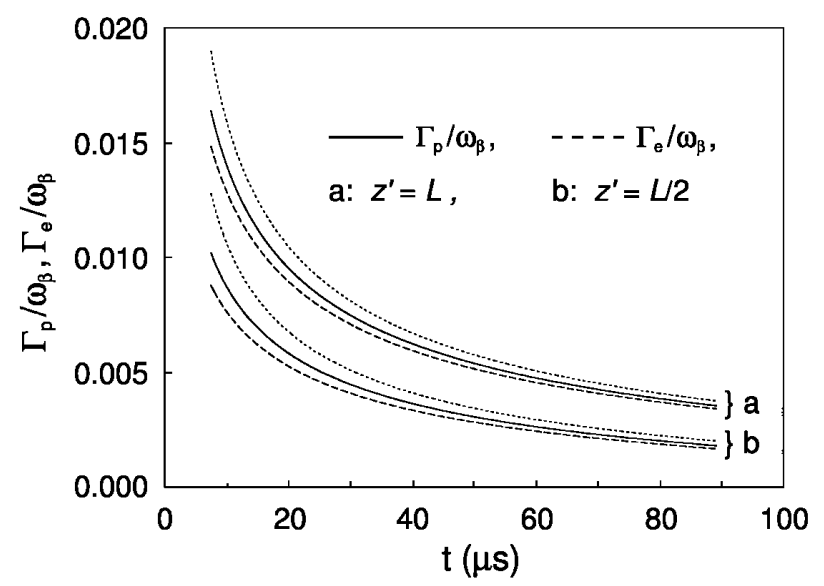

FIG. 3. The growth rates at the tail $\left(z^{\prime}=L\right)$ and the center $\left(z^{\prime}=L / 2\right)$ of the proton bunch are shown as functions of time. Here, $\Gamma_{p}$ and $\Gamma_{e}$ have been normalized to the betatron frequency $\omega_{\beta}$. The solid curves and the dashed curves are computed according to Eqs. (65) and (66), respectively. The dotted curves are computed using the asymptotic approximation in Eq. (67). The parameter values are described in the text. 


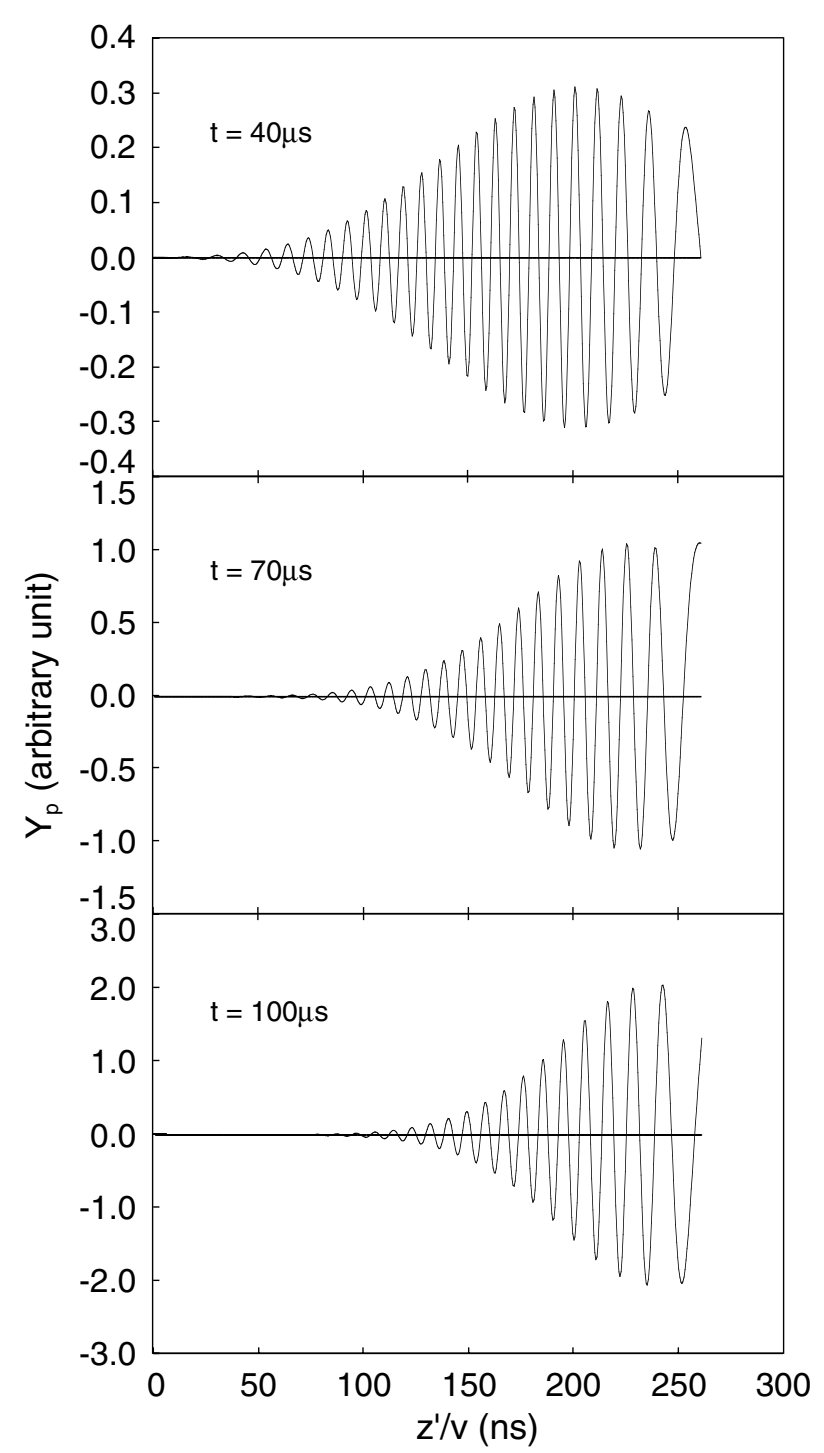

FIG. 4. The growing portion of the perturbed proton motion $Y_{p}$ as a function of $z^{\prime} / v$ at time $t=40 \mu \mathrm{s}, 70 \mu \mathrm{s}$, and $100 \mu \mathrm{s}$. Here, the value of $Y_{p}$ is computed using Eq. (55) with $\sigma_{p}=0$ and the parameter values described in the text. The proton bunch is $260 \mathrm{~ns}$ long. The vertical axis has arbitrary units.

The growing part of the perturbed electron motion $Y_{e}\left(z^{\prime}\right)$ is displayed in Fig. 5 for $\sigma_{e}=\pi / 2$ and the same time shown in Fig. 4. Note that the spatial damping due to the electron frequency spread is evident in Figs. 4 and 5 as a decrease of the oscillation amplitudes.

\section{CONCLUSIONS}

To model the $e-p$ interaction in a proton storage ring, we have derived the two-dimensional equations of motion for the centroids of the proton bunch and the electron cloud. The damping effect was incorporated in the centroid equations by considering Lorentzian and Gaussian distributions of the transverse oscillation frequencies of the particles. It was found that for both distributions, the

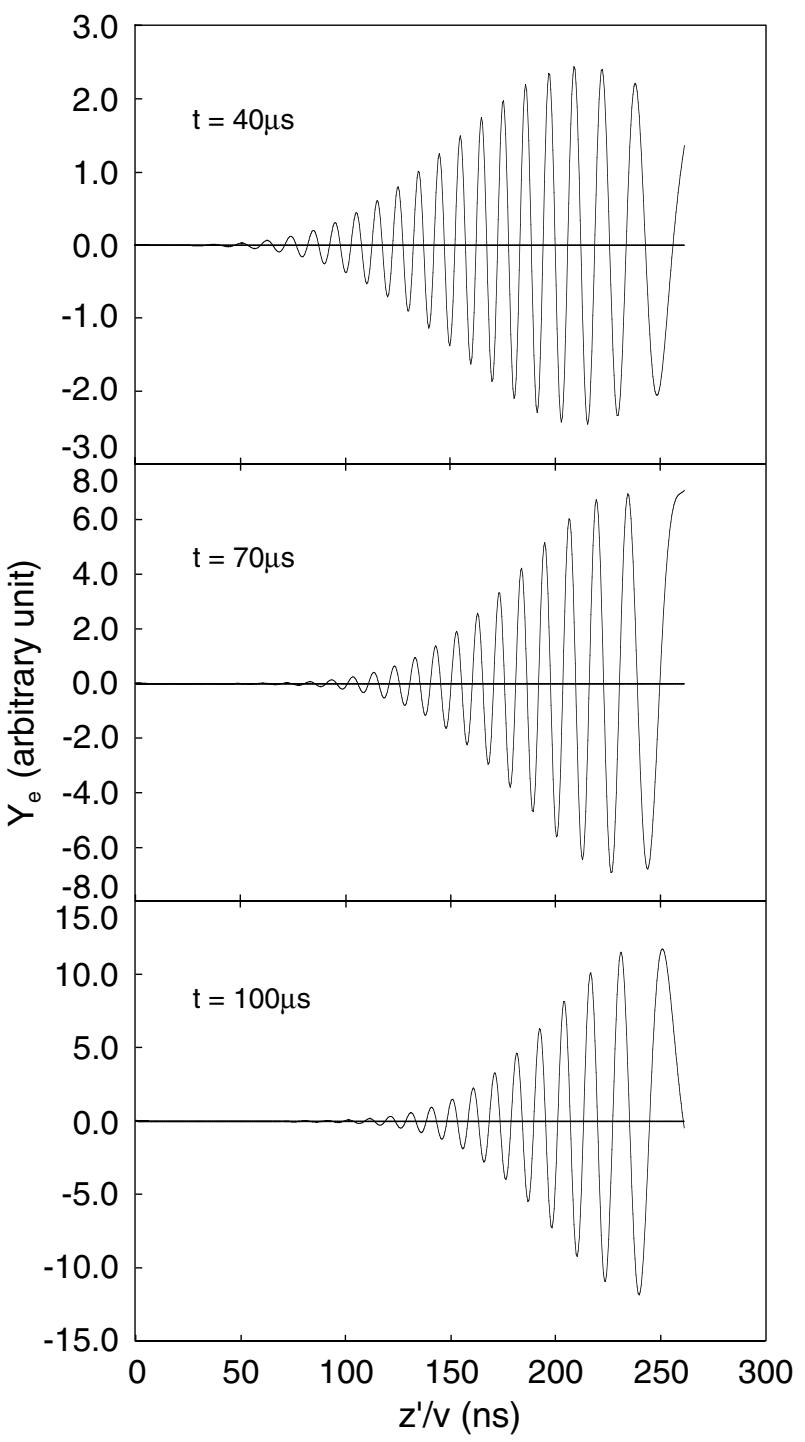

FIG. 5. The growing portion of the perturbed electron motion $Y_{e}$ as a function of $z^{\prime} / v$ at time $t=40 \mu \mathrm{s}, 70 \mu \mathrm{s}$, and $100 \mu \mathrm{s}$. Here, the value of $Y_{e}$ is computed using Eq. (58) with $\sigma_{e}=\pi / 2$ and the parameter values described in the text. The proton bunch is $260 \mathrm{~ns}$ long. The vertical axis has arbitrary units.

perturbed motion of the centroids damps exponentially. The exponential damping exponent is linear in time for the Lorentzian frequency distribution and quadratic in time for the Gaussian frequency distribution.

We have investigated the $e-p$ instability based on the centroid equations derived for a Lorentzian frequency distribution. The main features of the model studied in this work include the effects of finite proton bunch length, the one-pass interaction of the proton bunch with the background electrons, and the effects of nonuniform line densities. We have formulated an analytical approach to derive the asymptotic solution of the coupled linear centroid equations in the time domain for investigating the $e-p$ instability in a proton bunch propagating through 
a stationary electron background. We have also discussed the growth rate of the $e-p$ instability and derived a dispersion relation for the case of a uniform density electron background for frequency-domain studies. It was found that due to the one-pass electron-proton interaction, the unstable $e-p$ mode has an asymptotic growth that scales like $\exp \left\{\left[2 \omega_{\beta} J\left(z^{\prime}\right) t\right]^{1 / 2}\right\}$ for large $t$, where $J\left(z^{\prime}\right)$ is defined in Eq. (44). This result indicates that the growth rate of the $e-p$ instability is a function of both space and time. It also indicates that for a Lorentzian distribution of proton oscillation frequencies with nonzero spread, the instability will eventually be damped for sufficiently long times. It was shown that the electron oscillation frequency spread can cause spatial damping but not temporal damping in the beam frame, and that the asymptotic amplitude ratio between the proton oscillation and the electron oscillation is independent of the frequency spreads in particles. Numerical results obtained from applying the theory were presented by considering proton line densities with uniform and parabolic profiles.

\section{ACKNOWLEDGMENTS}

This research was supported by the Department of Energy.

\section{APPENDIX A: THE EXACT SOLUTIONS}

To derive the exact solutions for $Y_{p}\left(z^{\prime}, t\right)$ and $Y_{e}\left(z^{\prime}, t\right)$, we start with the approximation

$$
\hat{Y}\left(z^{\prime}, \omega\right) \approx \omega_{\beta}^{-3} \Phi\left(z^{\prime}\right) \exp \left[\frac{i \omega_{\beta}^{2} J\left(z^{\prime}\right)}{\omega^{2}-\omega_{\beta}^{2}}\right]
$$

obtained from Eqs. (37) and (42) in the main text, and the differential equation

$$
\frac{d^{2} \hat{Y}}{d z^{\prime 2}}+\frac{\Omega^{2}\left(z^{\prime}\right)}{v^{2}}\left[1+\frac{G\left(z^{\prime}\right)}{\omega^{2}-\omega_{\beta}^{2}}\right] \hat{Y}=0 .
$$

Equation (A2) can be derived by taking derivatives of Eq. (36) and making appropriate substitutions. The quantity $J\left(z^{\prime}\right)$ in Eq. (A1) is defined in Eq. (44). We will determine the solution of Eq. (A2) first. Then, $Y_{p}\left(z^{\prime}, t\right)$ will be derived by using Eqs. (24), (33), and (35). After that, $Y_{e}\left(z^{\prime}, t\right)$ will be deduced from Eq. (20). The exact solutions containing $\Psi\left(z^{\prime}\right)$ can be obtained by taking the complex conjugate of the results derived here.

Assuming the exact solution can be expressed as

$$
\hat{Y}\left(z^{\prime}, \omega\right)=\omega_{\beta}^{-3} Q\left(z^{\prime}, \omega\right) \Phi\left(z^{\prime}\right) \exp \left[\frac{i \omega_{\beta}^{2} J\left(z^{\prime}\right)}{\omega^{2}-\omega_{\beta}^{2}}\right],
$$

we substitute into Eq. (A2), which yields

$$
\frac{d^{2} Q}{d z^{\prime 2}}+\left(\frac{2}{\Phi} \frac{d \Phi}{d z^{\prime}}\right) \frac{d Q}{d z^{\prime}}+\left(\frac{2 i \omega_{\beta}^{2} d J / d z^{\prime}}{\omega^{2}-\omega_{\beta}^{2}}\right) \frac{d Q}{d z^{\prime}}-\left(\frac{\omega_{\beta}^{2} d J / d z^{\prime}}{\omega^{2}-\omega_{\beta}^{2}}\right)^{2} Q+\frac{1}{\omega^{2}-\omega_{\beta}^{2}}\left[2 i \omega_{\beta}^{2} \frac{d^{2} J}{d z^{\prime 2}}+\frac{\Phi \Psi}{v^{2} W} \frac{d\left(\Omega^{2} G\right)}{d z^{\prime}}\right] Q=0,
$$

where $W$ is defined in Eq. (30) as the Wronskian of $\Phi$ and $\Psi$.

Expanding $Q\left(z^{\prime}, \omega\right)$ as the infinite series

$$
Q\left(z^{\prime}, \omega\right)=\sum_{n=-\infty}^{\infty} \frac{a_{n}\left(z^{\prime}\right)}{\left(\omega^{2}-\omega_{\beta}^{2}\right)^{n}},
$$

we derive a sufficient condition for $Q\left(z^{\prime}, \omega\right)$ to be a solution of Eq. (A4). This gives

$$
\frac{d^{2} a_{n}}{d z^{\prime 2}}+\left(\frac{2}{\Phi} \frac{d \Phi}{d z^{\prime}}\right) \frac{d a_{n}}{d z^{\prime}}+2 i \omega_{\beta}^{2} \frac{d}{d z^{\prime}}\left(a_{n-1} \frac{d J}{d z^{\prime}}\right)+\frac{\Phi \Psi}{v^{2} W}\left[\frac{d\left(\Omega^{2} G\right)}{d z^{\prime}}\right] a_{n-1}-\omega_{\beta}^{4}\left(\frac{d J}{d z^{\prime}}\right)^{2} a_{n-2}=0 .
$$

A possible nontrivial solution for $a_{n}$ is

$$
\begin{gathered}
a_{m}=0, \quad \text { for } m<0, \\
a_{0}=1, \\
a_{1}=-\int^{z^{\prime}} \frac{d x}{\Phi^{2}(x)} \int^{x} \Phi^{2}(y)\left[2 i \omega_{\beta}^{2} \frac{d^{2} J}{d y^{2}}+\frac{\Phi \Psi}{v^{2} W} \frac{d\left(\Omega^{2} G\right)}{d y}\right] d y . \\
\vdots \\
a_{n}=-\int^{z^{\prime}} \frac{d x}{\Phi^{2}(x)} \int^{x} \Phi^{2}(y)\left\{2 i \omega_{\beta}^{2} \frac{d}{d y}\left(a_{n-1} \frac{d J}{d y}\right)+\frac{\Phi \Psi}{v^{2} W}\left[\frac{d\left(\Omega^{2} G\right)}{d y}\right] a_{n-1}-\omega_{\beta}^{4}\left(\frac{d J}{d y}\right)^{2} a_{n-2}\right\} d y,
\end{gathered}
$$

for $n \geq 2$. Therefore, the exact solution for $\hat{Y}\left(z^{\prime}, k\right)$ can be expressed as 


$$
\hat{Y}\left(z^{\prime}, \omega\right)=\omega_{\beta}^{-3} \Phi\left(z^{\prime}\right) \exp \left[\frac{i \omega_{\beta}^{2} J\left(z^{\prime}\right)}{\omega^{2}-\omega_{\beta}^{2}}\right] \sum_{n=0}^{\infty} \frac{a_{n}\left(z^{\prime}\right)}{\left(\omega^{2}-\omega_{\beta}^{2}\right)^{n}}
$$

where $a_{n}$ is defined in Eqs. (A8)-(A10). Then from Eqs. (24), (33), and (35) we obtain

$$
Y_{p}\left(z^{\prime}, t\right)=\frac{1}{\sqrt{2 \pi}} \xi\left(z^{\prime}\right) \Phi\left(z^{\prime}\right) e^{-\Delta_{p}\left(t-z^{\prime} / v\right)-\Delta_{e}\left(z^{\prime} / v\right)} \sum_{n=0}^{\infty} \frac{a_{n}}{\omega_{\beta}} \vartheta_{n},
$$

where $\xi\left(z^{\prime}\right)$ is defined in Eq. (16), and $\vartheta_{m}$ is the Fourier integral defined in Eq. (B1) and evaluated in Eq. (B10) in Appendix B. Thus, using Eq. (B10), we obtain

$$
\begin{aligned}
Y_{p}\left(z^{\prime}, t\right)= & -i \sqrt{2 \pi} \xi\left(z^{\prime}\right) \Phi\left(z^{\prime}\right) e^{-\Delta_{p}\left(t-z^{\prime} / v\right)-\Delta_{e}\left(z^{\prime} / v\right)} \sum_{n=0}^{\infty} \frac{a_{n}}{\left(2 \omega_{\beta}\right)^{2 n}} \sum_{m=1-n}^{\infty} \frac{1}{(m+n-1) !}\left(\frac{i u}{4 \alpha}\right)^{m} \\
& \times\left[e^{-i \alpha} I_{m}(u)-e^{i \alpha} J_{m}(u)\right] \frac{d^{m+n-1}}{d p^{m+n-1}}\left[\left.\frac{1}{p^{n}} \exp \left(\frac{-i u^{2}}{8 \alpha p}\right)\right|_{p=1},\right.
\end{aligned}
$$

where $J_{n}(x)$ and $I_{n}(x)$ are the Bessel function of the first kind and the modified Bessel function of the first kind, respectively, $a_{n}$ is defined in Eqs. (A8)-(A10), and $\alpha$ and $u$ are defined by

$$
\alpha=\omega_{\beta}\left(t-z^{\prime} / v\right)
$$

and

$$
u=\sqrt{2 \omega_{\beta} J\left(z^{\prime}\right)\left(t-z^{\prime} / v\right)}
$$

To find the exact solution for $Y_{e}\left(z^{\prime}, t\right)$, we first substitute Eq. (A12) into Eq. (20) in the main text. This gives

$$
Y_{e}\left(z^{\prime}, t\right)=\frac{-1}{\sqrt{2 \pi}} \Phi\left(z^{\prime}\right) e^{-\Delta_{p}\left(t-z^{\prime} / v\right)-\Delta_{e}\left(z^{\prime} / v\right)} \sum_{n=0}^{\infty} \frac{a_{n}}{\omega_{\beta}^{3}} \vartheta_{n-1} .
$$

Applying the result in Eq. (B10) to Eq. (A16), we obtain the exact solution for $Y_{e}\left(z^{\prime}, t\right)$ as

$$
\begin{aligned}
Y_{e}\left(z^{\prime}, t\right)= & i 8 \sqrt{2 \pi} \Phi\left(z^{\prime}\right) e^{-\Delta_{p}\left(t-z^{\prime} / v\right)-\Delta_{e}\left(z^{\prime} / v\right)} \sum_{n=0}^{\infty} \frac{a_{n}}{\left(2 \omega_{\beta}\right)^{2 n}} \sum_{m=2-n}^{\infty} \frac{1}{(m+n-2) !}\left(\frac{i u}{4 \alpha}\right)^{m} \\
& \times\left[e^{-i \alpha} I_{m}(u)-e^{i \alpha} J_{m}(u)\right] \frac{d^{m+n-2}}{d p^{m+n-2}}\left[\left.\frac{1}{p^{n-1}} \exp \left(\frac{-i u^{2}}{8 \alpha p}\right)\right|_{p=1} .\right.
\end{aligned}
$$

\section{APPENDIX B: FOURIER TRANSFORMATION}

Here, we perform the Fourier transformation

$$
\vartheta_{n}=\int_{-\infty}^{\infty} \frac{1}{\left(k^{2}-\omega_{\beta}^{2}\right)^{n}} \exp \left[i k\left(t-\frac{z^{\prime}}{v}\right)+\frac{i \omega_{\beta}^{2} J\left(z^{\prime}\right)}{k^{2}-\omega_{\beta}^{2}}\right] d k .
$$

Defining

we rewrite $\vartheta_{n}$ as

$$
\theta=i k\left(t-z^{\prime} / v\right)
$$

$$
\vartheta_{n}=i(-1)^{n+1}\left(t-z^{\prime} / v\right)^{2 n-1} \int_{-i \infty}^{i \infty} \frac{1}{\left(\theta^{2}+\alpha^{2}\right)^{n}} \exp \left[\theta-\frac{u^{2}}{4(\theta-i \alpha)}+\frac{u^{2}}{4(\theta+i \alpha)}\right] d \theta,
$$

where $\alpha$ and $u$ are defined in Eqs. (A14) and (A15), respectively. There are two poles in the integrand at $\theta=i \alpha$ and $\theta=-i \alpha$. Hence, from Eq. (B3) we obtain 


$$
\begin{aligned}
\vartheta_{n}= & 2 \pi(-1)^{n}\left(t-z^{\prime} / v\right)^{2 n-1} \operatorname{Res}_{\theta=-i a}\left\{\frac{1}{\left(\theta^{2}+\alpha^{2}\right)^{n}} \exp \left[\theta-\frac{u^{2}}{4(\theta-i \alpha)}+\frac{u^{2}}{4(\theta+i \alpha)}\right]\right\} \\
& +2 \pi(-1)^{n}\left(t-z^{\prime} / v\right)^{2 n-1} \operatorname{Res}_{\theta=i a}\left\{\frac{1}{\left(\theta^{2}+\alpha^{2}\right)^{n}} \exp \left[\theta-\frac{u^{2}}{4(\theta-i \alpha)}+\frac{u^{2}}{4(\theta+i \alpha)}\right]\right\},
\end{aligned}
$$

where we have applied the residue theorem of complex integration, and $\operatorname{Res}_{x=a}\{f(x)\}$ denotes the residue of $f(x)$ at $x=a$. We consider the residue at $\theta=-i \alpha$ first. Letting $s=\theta+i \alpha$ and employing the relation

we can expand the integrand as

$$
\exp \left(s+\frac{u^{2}}{4 s}\right)=\sum_{n=-\infty}^{\infty}\left(\frac{2 s}{u}\right)^{n} I_{n}(u)
$$

$$
\frac{1}{\left(\theta^{2}+\alpha^{2}\right)^{n}} \exp \left[\theta-\frac{u^{2}}{4(\theta-i \alpha)}+\frac{u^{2}}{4(\theta+i \alpha)}\right]=\frac{e^{-i a}}{s^{n}(s-2 i \alpha)^{n}} \exp \left[\frac{-u^{2}}{4(s-2 i \alpha)}\right] \sum_{m=-\infty}^{\infty}\left(\frac{2 s}{u}\right)^{m} I_{m}(u),
$$

where $I_{n}(x)$ is the modified Bessel function of the first kind. The pole is now at $s=0$, and

$$
s^{k} \exp \left[\frac{-u^{2}}{4(s-2 i \alpha)}\right]
$$

is analytic around $s=0$ for $k \geq 0$. Therefore, it follows that

$$
\begin{aligned}
& \operatorname{Res}_{\theta=-i a}\left\{\frac{1}{\left(\theta^{2}+\alpha^{2}\right)^{n}} \exp \left[\theta-\frac{u^{2}}{4(\theta-i \alpha)}+\frac{u^{2}}{4(\theta+i \alpha)}\right]\right\} \\
& =e^{-i \alpha} \sum_{m=-\infty}^{n-1}\left(\frac{2}{u}\right)^{m} I_{m}(u) \operatorname{Res}_{s=0}\left\{\frac{s^{m-n}}{(s-2 i \alpha)^{n}} \exp \left[\frac{-u^{2}}{4(s-2 i \alpha)}\right]\right\} \\
& =-e^{-i \alpha} \sum_{m=1-n}^{\infty}\left(\frac{-u}{2}\right)^{m} \frac{I_{m}(u)}{(m+n-1) !} \frac{d^{m+n-1}}{d r^{m+n-1}}\left\{\left.\frac{1}{(r+2 i \alpha)^{n}} \exp \left[\frac{u^{2}}{4(r+2 i \alpha)}\right]\right|_{r=0},\right.
\end{aligned}
$$

where $r=-s$.

By the same token, the residue at $\theta=i \alpha$ can be evaluated as

$$
\begin{aligned}
\operatorname{Res}_{\theta=i a}\left\{\frac{1}{\left(\theta^{2}+\alpha^{2}\right)^{n}} \exp \left[\theta-\frac{u^{2}}{4(\theta-i \alpha)}+\frac{u^{2}}{4(\theta+i \alpha)}\right]\right\}= & e^{i \alpha} \sum_{m=1-n}^{\infty}\left(\frac{-u}{2}\right)^{m} \frac{J_{m}(u)}{(m+n-1) !} \\
& \times \frac{d^{m+n-1}}{d r^{m+n-1}}\left\{\left.\frac{1}{(r+2 i \alpha)^{n}} \exp \left[\frac{u^{2}}{4(r+2 i \alpha)}\right]\right|_{r=0},\right.
\end{aligned}
$$

where $J_{n}(x)$ is the Bessel function of the first kind. Substituting Eqs. (B8) and (B9) and $r=2 i \alpha(p-1)$ into Eq. (B4), we obtain

$$
\vartheta_{n}=\frac{-2 \pi i}{\left(2 \omega_{\beta}\right)^{2 n-1}} \sum_{m=1-n}^{\infty} \frac{1}{(m+n-1) !}\left(\frac{i u}{4 \alpha}\right)^{m}\left[e^{-i \alpha} I_{m}(u)-e^{i \alpha} J_{m}(u)\right] \frac{d^{m+n-1}}{d p^{m+n-1}}\left[\left.\frac{1}{p^{n}} \exp \left(\frac{-i u^{2}}{8 \alpha p}\right)\right|_{p=1} .\right.
$$

The derivative with respect to $p$ in Eq. (B10) can be expressed as a double summation of finite series by using Leibniz' formula, but we will omit this step for brevity.

When $n=0$, we obtain

$$
\begin{aligned}
\vartheta_{0}= & \frac{\pi u e^{-i J\left(z^{\prime}\right) / 4}}{t-z^{\prime} / v}\left[e^{-i \alpha} I_{1}(u)-e^{i \alpha} J_{1}(u)\right]-\frac{\pi e^{-i J\left(z^{\prime}\right) / 4}}{t-z^{\prime} / v}\left(\frac{u^{4}}{32 \alpha^{2}}\right)\left[e^{-i \alpha} I_{2}(u)-e^{i \alpha} J_{2}(u)\right] \\
& -4 \pi i \omega_{\beta} \sum_{m=3}^{\infty} \frac{1}{(m-1) !}\left(\frac{i u}{4 \alpha}\right)^{m}\left[e^{-i \alpha} I_{m}(u)-e^{i \alpha} J_{m}(u)\right] \frac{d^{m-1}}{d p^{m-1}}\left[\left.\frac{1}{p^{n}} \exp \left(\frac{-i u^{2}}{8 \alpha p}\right)\right|_{p=1} .\right.
\end{aligned}
$$

For $u^{2}<\alpha$ or $u^{2} \approx \alpha$, we therefore obtain the following approximation for $\vartheta_{0}$ by neglecting all of the $m \geq 3$ terms in Eq. (B11):

$$
\vartheta_{0} \approx \frac{\pi u e^{-i J\left(z^{\prime}\right) / 4}}{t-z^{\prime} / v}\left\{e^{-i \alpha}\left[I_{1}(u)-\frac{u^{3}}{32 \alpha^{2}} I_{2}(u)\right]-e^{i \alpha}\left[J_{1}(u)-\frac{u^{3}}{32 \alpha^{2}} J_{2}(u)\right]\right\} .
$$


[1] Proceedings of the ECLOUD'02, Geneva, edited by G. Rumolo and F. Zimmermann (CERN, Geneva, 2002) (CERN Report No. CERN-2002-001, 2002).

[2] Proceedings of the International Workshop on TwoStream Instabilities in Particle Accelerator and Storage Rings, 2001, KEK, Tsukuba-shi, Japan, accessible at the web site http://conference.kek.jp/two-stream/.

[3] Proceedings of the 2001 Particle Accelerator Conference, Chicago, IL, edited by P. Lucas and S. Webber (IEEE, Piscataway, NJ, 2001).

[4] Proceedings of the ICFA 8th Beam Dynamics Mini-Workshop on Two-Stream Instabilities in Particle Accelerators and Storage Rings, 2000, edited by K. Harkay and R. J. Macek, accessible at the web site http://www.aps.anl.gov/conferences/icfa/ two-stream.html.

[5] Proceedings of the Workshop in Instabilities of High Intensity Hadron Beams in Rings, edited by T. Roser and S. Y. Zhang, AIP Conf. Proc. No. 496 (AIP, New York, 1999).

[6] Proceedings of the Santa Fe Workshop on Electron Effects, edited by T. F. Wang and A. Jason (LANL, Los Alamos, NM, 1998) (LANL Report No. LA-UR-981601, 1998).

[7] R. J. Macek, in Proceedings of the ECLOUD'02, Geneva (Ref. [1]), p. 259.

[8] M. Pivi and M. A. Furman, in Proceedings of the European Particle Accelerator Conference, Paris, 2002 (to be published).

[9] M. Pivi and M. A. Furman, in Proceedings of the ECLOUD'02, Geneva (Ref. [1]), p. 137.

[10] R. J. Macek, A. Browman, D. H. Fitzgerald, R. McCrady, F. Merill, M. Plum, T. Spickermann, T.S. Wang, J. Griffin, K. Y. Ng, D. Wildman, K. Harkay, R. Kustom, and R. A. Rosenberg, in Proceedings of the 2001 Particle Accelerator Conference, Chicago, IL (Ref. [3]), Vol. 1, p. 688.

[11] T. F. Wang, P. J. Channell, R. J. Macek, and R. C. Davidson, in Proceedings of the 2001 Particle Accelerator Conference, Chicago, IL (Ref. [3]), Vol. 1, p. 704.

[12] H. Qin, R. C. Davidson, W.W. Lee, and E. Startsev, in Proceedings of the 2001 Particle Accelerator Conference, Chicago, IL (Ref. [3]), Vol. 1, p. 696.

[13] M. A. Furman and M. Pivi, in Proceedings of the 2001 Particle Accelerator Conference, Chicago, IL (Ref. [3]), Vol. 1, p. 707.

[14] R. J. Macek, A. Browman, D. H. Fitzgerald, R. McCrady, M. Plum, and T. Spickermann, in Proceedings of the ICANS-XV, Tokaimura, Japan, 2001, edited by J. Suzuki and S. Itoh (JAERI, Tokai-mura, Japan, 2001) (JAERI Report No. JAERI-Conf 2001-002, 2001), Vol. I, p. 229.

[15] H. Qin, R. C. Davidson, and W.W. Lee, Phys. Rev. ST Accel. Beams 3, 084401 (2000); 4, 109901 (2000).

[16] M. Blaskiewicz, in Proceedings of the EPAC 2000, Vienna, edited by J. L. Laclar, W. Mitaroff, Ch. Petit-
Jean-Genaz, J. Poole, and M. Regler (Austrian Academy of Science Press, Vienna, 2000), p. 1110.

[17] R. C. Davidson, H. Qin, P. H. Stoltz, and T. F. Wang, Phys. Rev. ST Accel. Beams 2, 054401 (1999).

[18] R. C. Davidson, H. Qin, W.W. Lee, and T. F. Wang, in Proceedings of the 1999 Particle Accelerator Conference, New York, edited by A. Luccio and W. MacKay (IEEE, Piscataway, NJ, 1999), Vol. 3, p. 1623.

[19] H. Qin, R. C. Davidson, and W.W. Lee, in Proceedings of the 1999 Particle Accelerator Conference, New York (Ref. [18]), Vol. 3, p. 1628.

[20] T.F. Wang, in Proceedings of the Workshop in Instabilities of High Intensity Hadron Beams in Rings (Ref. [5]), p. 305.

[21] M. Blaskiewicz, in Proceedings of the Workshop in Instabilities of High Intensity Hadron Beams in Rings (Ref. [5]), p. 321.

[22] D. Neuffer, E. Colton, D. Fitzgerald, T. Hardek, R. Hutson, R. Macek, M. Plum, H. Thiessen, and T.-S. Wang, Nucl. Instrum. Methods Phys. Res., Sect. A 321, 1 (1992).

[23] D. Neuffer, E. Colton, G. Swain, H. A. Thiessen, B. Blind, A. Jason, G. Lawrence, R. Shafer, T. Hardek, J. Hurd, R. Macek, and M. Plum, Part. Accel. 23, 133 (1988).

[24] T. F. Wang, A. Pisent, and D. V. Neuffer, in Proceedings of the Advanced Hadron Facility Accelerator Design Workshop, edited by H. A. Thiessen (LANL, Los Alamos, NM, 1989) (LANL Report No. LA-11684-C, 1990), p. 818.

[25] R. C. Davidson and H. Qin, Physics of Intense Charged Particle Beams in High Energy Accelerators (Imperial College Press and World Scientific Publishing Co., London, 2001), Chap. 10.

[26] L. J. Laslett, A. M. Sessler, and D. Möhl, Nucl. Instrum. Methods 121, 517 (1974).

[27] D. G. Koshkarev and P. R. Zenkevich, Part. Accel. 3, 1 (1972).

[28] E. Keil and B. Zotter, CERN Internal Note No. CERNISR-TH/71-58, 1971.

[29] B. V. Chirikov, Atomnaya Énergiya 19, No. 3, 239 (1965).

[30] The perturbed transverse field due to a small displacement of a beam has been discussed in many studies. Examples can be found in E. Regenstreif, CERN Internal Report No. CERN/PS/DL 76-2, 1976; T. F. Wang, LANL Report No. LA-UR-98-5226, 1998.

[31] For example, see S. Y. Lee, Accelerator Physics (World Science Publishing Co. Pte. Ltd., River Edge, NJ, 1999), p. 199.

[32] T.F. Wang, LANL PSR Technical Note No. 02-001, LANSCE1:02-035, 2002.

[33] M. Abramowitz and I. A. Stegun, in Handbook of Mathematical Functions, Applied Mathematics Series, Natl. Bur. Stand. (U.S.) Circular No. 55 (U.S. GPO, Washington, DC, 1964). 